\title{
OSCILLATORY AND PERIODIC SOLUTIONS TO A DIFFUSION EQUATION OF NEUTRAL TYPE
}

\author{
JOSEPH WIENER and WILLIAM HELLER
}

(Received 30 October 1997)

\begin{abstract}
We examine a PDE with piecewise constant time delay. The equation is of neutral type since it contains the derivative $u_{t}$ at different values of the $t$-argument. Furthermore, the argument deviation changes its sign within intervals of unit length, so that the given PDE is alternately of retarded and advanced type. It is shown that the argument deviation generates, under certain conditions, oscillations of the solutions, which is an impossible phenomenon for the corresponding equation without delay. Of special interest is the appearance of periodic solutions as well as solutions asymptotically approaching closed curves which are not solutions of the equation studied.
\end{abstract}

Keywords and phrases. Partial differential equations, piecewise constant argument, piecewise constant delay, boundary value problem, periodic solution, oscillatory solution.

1991 Mathematics Subject Classification. 35A05, 35B25, 35L10, 34K25.

1. Introduction. The paper continues our earlier work on boundary value problems (BVP) for partial differential equations with piecewise constant argument (EPCA) which was initiated in [1]. These equations appear in an attempt to extend the theory of functional differential equations to systems with discontinuous argument deviations. EPCA also arise in the process of replacing some terms of a differential equation by their piecewise constant approximations. Thus, the equation

$$
u_{t}(x, t)=a^{2} u_{x x}(x, t)-b u(x, t)
$$

describes heat flow in a rod with both diffusion $a^{2} u_{x x}$ along the rod and heat loss (or gain) across the lateral sides of the rod. Measuring the lateral heat change at discrete moments of time leads to the equation with piecewise continuous delay

$$
u_{t}(x, t)=a^{2} u_{x x}(x, t)-b u(x,[t]),
$$

which was investigated in [1]. Here [·] designates the greatest integer function and $(x, t) \in[0,1] \times[0, \infty)$. The equation

$$
u_{t}(x, t)=a^{2} u_{x x}(x, t)-b u\left(x,\left[t+\frac{1}{2}\right]\right),
$$

with the boundary conditions

$$
u(0, t)=0, \quad u(1, t)=0,
$$

and the initial condition

$$
u(x, 0)=u_{0}(x),
$$

was investigated in [2]. 
A function $u(x, t)$ is said to be a solution of the above BVP if it satisfies the conditions

(i) $u(x, t)$ is continuous in $G=[0,1] \times[0, \infty)$;

(ii) $u_{t}$ and $u_{x x}$ exist and are continuous in $G$, with the possible exception of the points $(x, n+1 / 2)$, where one-sided derivatives exist $(n=0,1,2, \ldots)$;

(iii) $u(x, t)$ satisfies equation (1.3) in $G$, with the possible exception of the points $(x, n+1 / 2)$, and conditions (1.4) and (1.5).

Equation (1.3) is of considerable interest since the argument deviation

$$
\tau(t)=t-\left[t+\frac{1}{2}\right]
$$

changes sign in each interval $(n-1 / 2, n+1 / 2)$ with integer $n$. Indeed, $\tau(t)<0$ for $n-1 / 2 \leq t<n$ and $\tau(t)>0$ for $n<t<n+1 / 2$, which means that equation (1.3) is alternately of advanced and retarded type. The purpose of this paper is to explore the influence of terms with piecewise constant time on the behavior of the solutions, especially their oscillatory properties, for partial differential equations of neutral type.

2. A neutral system of EPCA. Consider the BVP consisting of the equation

$$
U_{t}(x, t)=A U_{x x}(x, t)+B U_{t}\left(x,\left[t+\frac{1}{2}\right]\right),
$$

the boundary condition

$$
U(0, t)=U(1, t)=0
$$

and the initial condition

$$
U(x, 0)=U_{0}(x) .
$$

Here, $U(x, t)$ and $U_{0}(x)$ are real $m \times m$ matrices, $A$ and $B$ are real constant $m \times m$ matrices and $[\cdot]$ denotes the greatest integer function. Equation (2.1) is of neutral type since it includes the derivative $U_{t}$ at different values of $t$.

Looking for a solution in the form

$$
U(x, t)=T(t) X(x)
$$

gives

$$
T^{\prime}(t) X(x)=A T(t) X^{\prime \prime}(x)+B T^{\prime}\left(\left[t+\frac{1}{2}\right]\right) X(x)
$$

whence

$$
\left(T^{\prime}(t)-B T^{\prime}\left(\left[t+\frac{1}{2}\right]\right)\right) X(x)=A T(t) X^{\prime \prime}(x)
$$

and

$$
T^{-1}(t) A^{-1}\left(T^{\prime}(t)-B T^{\prime}\left(\left[t+\frac{1}{2}\right]\right)\right)=X^{\prime \prime}(x) X^{-1}(x)=-P^{2},
$$

which generates the BVP

$$
X^{\prime \prime}(x)+P^{2} X(x)=0, \quad X(0)=X(1)=0,
$$

and the equation with piecewise constant argument

$$
T^{\prime}(t)=-A T(t) P^{2}+B T^{\prime}\left(\left[t+\frac{1}{2}\right]\right) .
$$


The general solution of equation (2.8) is

$$
X(x)=\cos (x P) C_{1}+\sin (x P) C_{2},
$$

where

$$
\cos (x P)=\sum_{n=0}^{\infty} \frac{(-1)^{n} x^{2 n} P^{2 n}}{(2 n) !}, \quad \sin (x P)=\sum_{n=0}^{\infty} \frac{(-1)^{n} x^{2 n+1} P^{2 n+1}}{(2 n+1) !},
$$

and $C_{1}, C_{2}$ are arbitrary constant matrices. From $X(0)=0$, we conclude that $C_{1}=0$, and the condition $X(1)=0$ enables us to choose $\sin P=0$ (although this is not the necessary consequence of the equation $\left.(\sin P) C_{2}=0\right)$. This can be written as

$$
e^{i P}-e^{-i P}=0, \quad e^{2 i P}=I .
$$

Assuming that all the eigenvalues $p_{1}, p_{2}, \ldots, p_{m}$ of $P$ are distinct and

$$
S^{-1} P S=D=\operatorname{diag}\left(p_{1}, p_{2}, \ldots, p_{m}\right),
$$

we have

$$
\exp \left(2 i S D S^{-1}\right)=I, \quad S e^{2 i D} S^{-1}=I, \quad e^{2 i D}=I .
$$

Therefore,

$$
D=\operatorname{diag}\left(\pi j_{1}, \pi j_{2}, \ldots, \pi j_{m}\right),
$$

where the $j_{k}$ are integers, and

$$
\begin{gathered}
P=S D S^{-1}, \\
P^{2}=S D^{2} S^{-1}=S \operatorname{diag}\left(\pi^{2} j_{1}^{2}, \pi^{2} j_{2}^{2}, \ldots, \pi^{2} j_{m}^{2}\right) S^{-1}, \\
\sin (x P)=S \sin (x D) S^{-1}=S \operatorname{diag}\left(\sin \left(\pi j_{1} x\right), \ldots, \sin \left(\pi j_{m} x\right)\right) S^{-1} .
\end{gathered}
$$

Furthermore, we can put

$$
P_{j}=\operatorname{diag}(\pi(m(j-1)+1), \ldots, \pi m j), \quad j=1,2, \ldots
$$

in (2.8) and obtain the following result.

THEOREM 2.1. There exists an infinite sequence of matrix eigenfunctions for BVP (2.8)

$$
X_{j}(x)=\sqrt{2} \operatorname{diag}(\sin (\pi(m(j-1)+1) x), \ldots, \sin (\pi m j x)), \quad j=1,2, \ldots
$$

which is complete and orthonormal in the space $L^{2}[0,1]$ of $m \times m$ matrices, that is,

$$
\int_{0}^{1} X_{j}(x) X_{k}(x) d x= \begin{cases}0, & j \neq k, \\ I, & j=k\end{cases}
$$

where I is the identity matrix.

Note that the matrices $S X_{j}(x) S^{-1}$ satisfy Theorem 2.1 for any nonsingular $S$.

THEOREM 2.2. Let $E(t)$ be the solution of the problem

$$
T^{\prime}(t)=-A T(t) P^{2}, \quad T(0)=I
$$


and let

$$
\begin{gathered}
M(t)=I-(E(t)-I) A^{-1}(B-I)^{-1} A, \\
M_{0}=M^{-1}\left(-\frac{1}{2}\right) M\left(\frac{1}{2}\right) .
\end{gathered}
$$

If the matrices $A, B-I$, and $M(-1 / 2)$ are nonsingular, then equation (2.9), with the initial condition $T(0)=C_{0}$, has on $[0, \infty)$ a unique solution

$$
T(t)=M\left(t-\left[t+\frac{1}{2}\right]\right) M_{0}^{[t+1 / 2]} C_{0} .
$$

Proof. On the interval $n-1 / 2 \leq t<n+1 / 2$, where $n \geq 0$ is an integer, equation (2.9) turns into

$$
T^{\prime}(t)=-A T(t) P^{2}+B T^{\prime}(n),
$$

with the general solution

$$
T(t)=E(t-n) C+A^{-1} B T^{\prime}(n) P^{-2} .
$$

At $t=n$, we find from (2.26) that

$$
B T^{\prime}(n)=T^{\prime}(n)+A T(n) P^{2},
$$

and substituting in (2.27), we get

$$
T(t)=E(t-n) C+A^{-1} T^{\prime}(n) P^{-2}+T(n) .
$$

Furthermore, from (2.26) we have

$$
T^{\prime}(n)=-A T(n) P^{2}+B T^{\prime}(n),
$$

whence

$$
T^{\prime}(n)=(B-I)^{-1} A T(n) P^{2} .
$$

At $t=n$, we find from (2.29) that

$$
C=-A^{-1} T^{\prime}(n) P^{-2}
$$

and

$$
T(t)=M(t-n) T(n),
$$

where $M(t)$ is defined in (2.23). Letting $t \rightarrow n+1 / 2$ from the left gives

$$
T\left(n+\frac{1}{2}\right)=M\left(\frac{1}{2}\right) T(n) .
$$

Furthermore, on the interval $n+1 / 2 \leq t<n+3 / 2$, we have

$$
T(t)=M(t-n-1) T(n+1),
$$

and letting $t \rightarrow n+1 / 2$ from the right yields

$$
T\left(n+\frac{1}{2}\right)=M\left(-\frac{1}{2}\right) T(n+1),
$$

that is,

$$
M\left(-\frac{1}{2}\right) T(n+1)=M\left(\frac{1}{2}\right) T(n) .
$$


From here,

$$
T(n+1)=M_{0} T(n)
$$

and

$$
T(n)=M_{0}^{n} T(0),
$$

where the matrix $M_{0}$ is given in (2.24). Substituting this expression in (2.33) proves (2.25).

3. A scalar EPCA of parabolic type. We examine the scalar version of the problem of the previous section and catalog the behavior of the solutions. To be specific, we examine the boundary value problem (BVP) for the equation with piecewise constant argument (EPCA)

$$
u_{t}(x, t)=a^{2} u_{x x}(x, t)+b u_{t}\left(x,\left[t+\frac{1}{2}\right]\right),
$$

with homogeneous boundary conditions

$$
u(0, t)=0, \quad u(1, t)=0,
$$

and initial condition

$$
u(x, 0)=u_{0}(x)
$$

Note that we have taken $A=a^{2}$ and $B=b$ in (2.1). If we let $P=\lambda$, separation of variables yields the scalar analog of equation (2.8), namely,

$$
X^{\prime \prime}+\lambda^{2} X=0, \quad X(0)=X(1)=0,
$$

with the orthonormal basis of solutions

$$
X_{j}(x)=\sqrt{2} \sin \lambda_{j} x, \quad \lambda_{j}=\pi j, j=1,2,3, \ldots
$$

on $[0,1]$. The scalar version of equation (2.9) is

$$
T_{j}^{\prime}(t)=-a^{2} \lambda_{j}^{2} T_{j}(t)+b T_{j}^{\prime}\left(\left[t+\frac{1}{2}\right]\right),
$$

where $T_{j}$ is the solution corresponding to $\lambda_{j}$. Since (3.1) is a particular case of (2.1), with $A=a^{2}$ and $B=b$, problem (2.22) becomes

$$
T_{j}^{\prime}(t)=-a^{2} \lambda_{j}^{2} T_{j}(t), \quad T_{j}(0)=1
$$

whose solution is

$$
E_{j}(t)=e^{-d_{j} t},
$$

where $d_{j}=a^{2} \lambda_{j}^{2}$. Then, by virtue of (2.23) and (2.24), it follows that

$$
M_{j}(t)=\frac{b-e^{-d_{j} t}}{b-1}
$$

and

$$
M_{0 j}=\frac{b-e^{-d_{j} / 2}}{b-e^{d_{j} / 2}} .
$$


Substituting this into (2.25) produces the solution of (3.6)

$$
T_{j}(t)=\left(\frac{b-e^{-d_{j} / 2}}{b-e^{d_{j} / 2}}\right)^{[t+1 / 2]}\left(\frac{b-e^{-d_{j}(t-[t+1 / 2])}}{b-1}\right) T_{j}(0) .
$$

The value $T_{j}(0)$ can be obtained from the initial condition. This follows when we write the solution $u(x, t)$ to (3.1) as a superposition of the functions $X_{j}$ and $T_{j}$ giving

$$
u(x, t)=\sum_{j=1}^{\infty} T_{j}(t) \sqrt{2} \sin (\pi j x) .
$$

When $t=0$, we have

$$
u(x, 0)=u_{0}(x)=\sum_{j=1}^{\infty} T_{j}(0) \sqrt{2} \sin (\pi j x) .
$$

Hence, $T_{j}(0)$ is simply the Fourier coefficient of $u_{0}(x)$, i.e.,

$$
T_{j}(0)=\hat{u}_{0}(j)=\sqrt{2} \int_{0}^{1} u_{0}(x) \sin (\pi j x) d x .
$$

With this description, we can now summarize in the theorems below the behavior of the solutions to problem (3.1) and illustrate the far more complicated behavior of solutions to EPCA problems as compared to problems without a time delay.

THEOREM 3.1. The time functions $T_{j}(t)$ in the separation of variables solution (3.12) to the boundary value problem (3.1) with $b<0$ tend to zero monotonically as $t \rightarrow \infty$. (See Figure 3.1.)

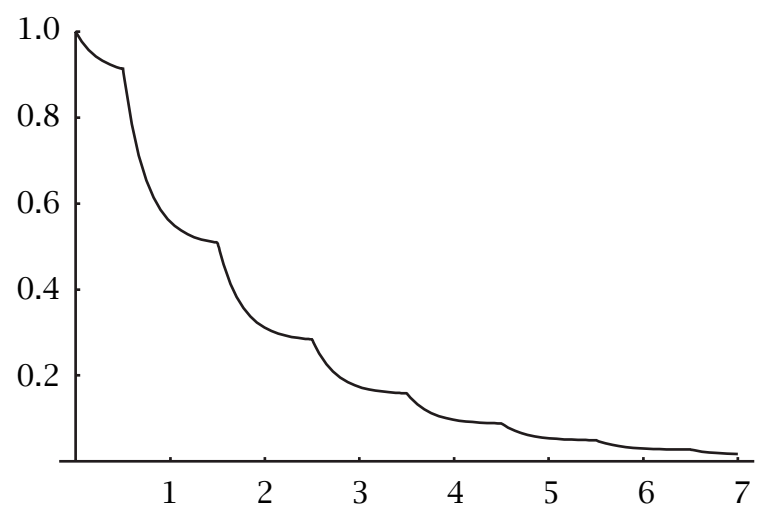

FiguRE 3.1. $T_{j}(t)$ for $b(t)=b, b=-0.9, j=2, a=1 / \pi$.

Proof. For the proof of Theorem 3.1 as well as the proofs of the next two theorems, we must examine the ratio

$$
r(j) \equiv \frac{b-e^{-d_{j} / 2}}{b-e^{d_{j} / 2}}
$$

which appears in (3.11). Also, note that the other factor in (3.11),

$$
H_{j}(t) \equiv\left(\frac{b-e^{-d_{j}(t-[t+1 / 2])}}{b-1}\right)
$$


is a function of period 1 and is monotone on each interval of the form [ $n-1 / 2$, $n+1 / 2), n=0,1,2, \ldots$.

If $b<0$, the ratio satisfies the inequality $0<r(j)<1$. The left inequality is obvious. The right inequality holds because if $r(j) \geq 1$, then we have the impossibility $e^{d_{j}} \leq 1$. Furthermore, $H_{j}(t)$ is monotonically decreasing on each interval $[n-1 / 2, n+1 / 2)$, so $T_{j}(t) \rightarrow 0$ monotonically as $t \rightarrow \infty$. See Figure 3.1 for a typical example of a $T_{j}$ function for $b<0$. Note the discontinuities in the derivatives at the points $n+1 / 2$, $n=0,1,2,3, \ldots$ where we have jumps in the piecewise constant argument $[t+1 / 2]$. In this case, the corresponding solutions to the nondelay equation (see (3.26) and (3.27) below) are also monotonically decaying to zero.

THEOREM 3.2. For $0<b<1$, the behavior of the time functions $T_{j}(t)$ in (3.12) depends on the relative values of $j$ and

$$
W \equiv \sqrt{\frac{-2 \ln b}{a^{2} \pi^{2}}} .
$$

Specifically,

(a) If $j<W$, then $T_{j}(t) \rightarrow 0$ monotonically as $t \rightarrow \infty$.

(b) If $j=W, T_{j}(t)=0$ for $t \geq 1 / 2$ and $T_{j}(t) \rightarrow 0$ monotonically as $t \rightarrow 1 / 2^{-}$on $[0,1 / 2]$.

(c) If $j>W$, then $T_{j}(t) \rightarrow 0$ as $t \rightarrow \infty$, but oscillates (crosses the time axis in each interval $[n-1 / 2, n+1 / 2), n=0,1,2, \ldots)$. (See Figure 3.2.)

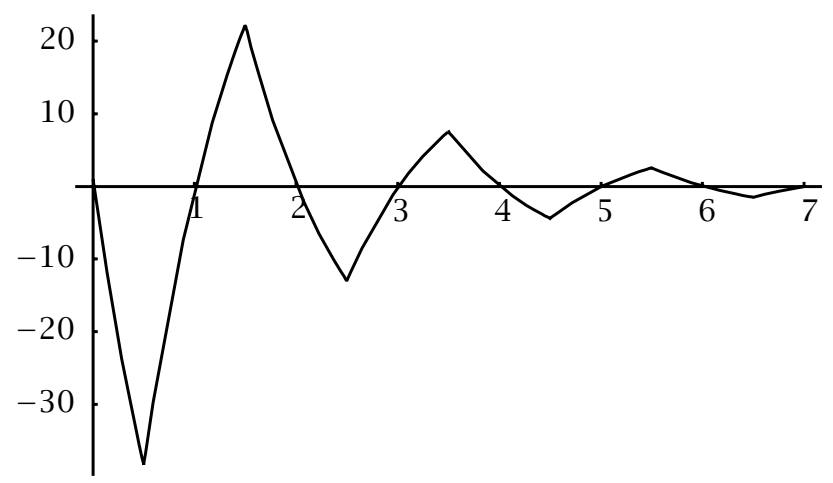

FIGURE 3.2. $T_{j}(t)$ for $b(t)=b, b=0.99, j=1, a=1 / \pi$.

Proof. The ratio defined above satisfies $-1<r(j)<1$ for $0<b<1$, as can be seen by arguments similar to those used in the proof of Theorem 3.1. This means that the solutions $T_{j}$ dampen with increasing time. If, in addition, $j<W$, then $0<r(j)<1$ so that the solutions $T_{j}$ monotonically dampen to zero.

On the other hand, if $j>W$, the ratio satisfies $-1<r(j)<0$ so that solutions dampen to zero and oscillate. See Figure 3.2. 
Finally, for $j=W$, we have the equivalent condition

$$
b-e^{-d_{j} / 2}=0
$$

and so $r(j)=0$ for this value of $j$. By equation (3.11), we see that $T_{j}(t)=0$ for all $t \geq 1 / 2$. On the initial interval $[0,1 / 2], T_{j}(t)$ goes to zero monotonically because $H_{j}(t)$ is monotone on $[0,1 / 2)$.

The $T_{j}$ functions for the nondelay equation, given below in (3.27), monotonically dampen to zero for all $j$ and so do not oscillate when $0<b<1$.

THEOREM 3.3. For $b>1$, the behavior of the time functions $T_{j}(t)$ in (3.12) depends on the value of $j$ and the numbers

$$
Q \equiv \sqrt{\frac{2 \ln b}{a^{2} \pi^{2}}}, \quad R \equiv \sqrt{\frac{2 \cosh ^{-1}(b)}{a^{2} \pi^{2}}} .
$$

(a) If $j<Q$, then $T_{j}(t)$ grows monotonically and without bound as $t \rightarrow \infty$ provided $T_{j}(0) \neq 0$. (See Figure 3.3.)

(b) If $j=Q$, then $T_{j}(t)=0$ for all $t \geq 0$ provided $u_{0}(x)$ is orthogonal to $\sqrt{2} \sin (\pi j x)$; otherwise the separation of variables method does not provide a solution.

(c) If $Q<j<R$, then $T_{j}(t)$ grows without bound and oscillates provided $T_{j}(0) \neq 0$. (See Figure 3.4.)

(d) If $j=R$, then $T_{j}(t)$ is periodic and oscillating for all $t \geq 0$. (See Figure 3.5.)

(e) If $j>R$, then $T_{j}(t) \rightarrow 0$ as $t \rightarrow \infty$, but oscillates. (See Figure 3.6.)

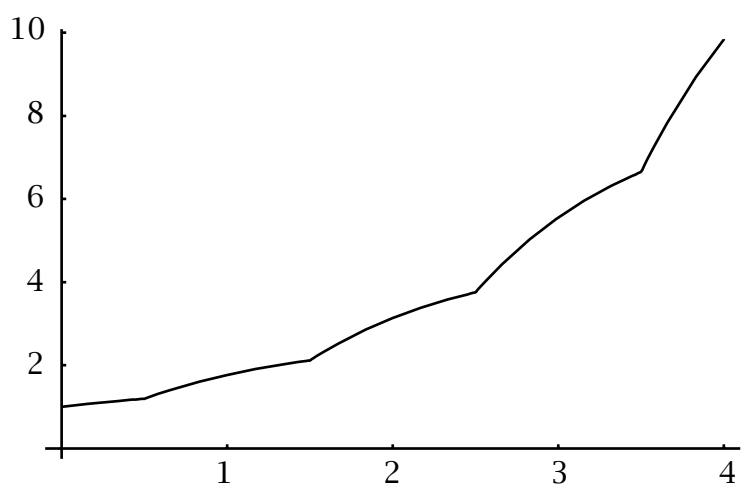

FIGURE 3.3. $T_{j}(t)$ for $b(t)=b, b=3, j=1, a=1 / \pi$.

Proof. If $j<Q$, the ratio $r(j)>1$ so that solutions $T_{j}$ grow monotonically and without bound provided $T_{j}(0)=\hat{u}_{0}(j) \neq 0$, but do not oscillate. See Figure 3.3 for an illustration of this.

When $Q<j<R$, the ratio $r(j)<-1$. So solutions $T_{j}$ grow without bound and oscillate provided $T_{j}(0)=\hat{u}_{0}(j) \neq 0$. See Figure 3.4.

If $j=R$, then $r(j)=-1$ so that we have oscillating, periodic solutions with period 2 . See Figure 3.5. 


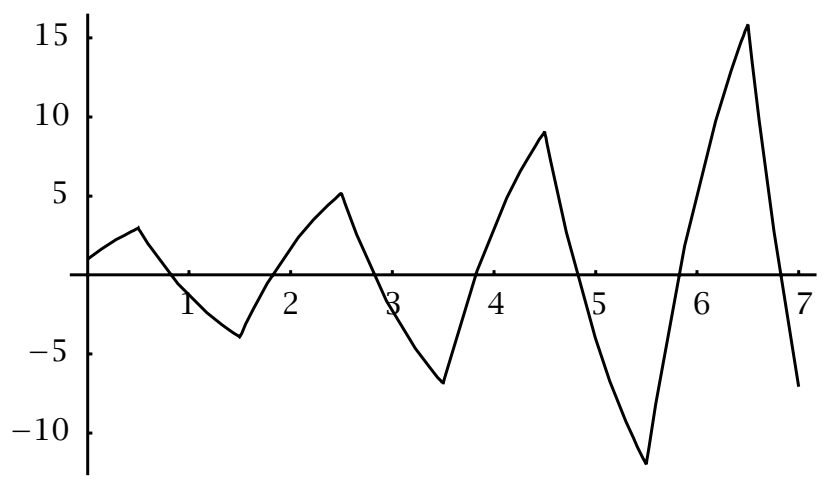

FIGURE 3.4. $T_{j}(t)$ for $b(t)=b, b=1.2, j=1, a=1 / \pi$.

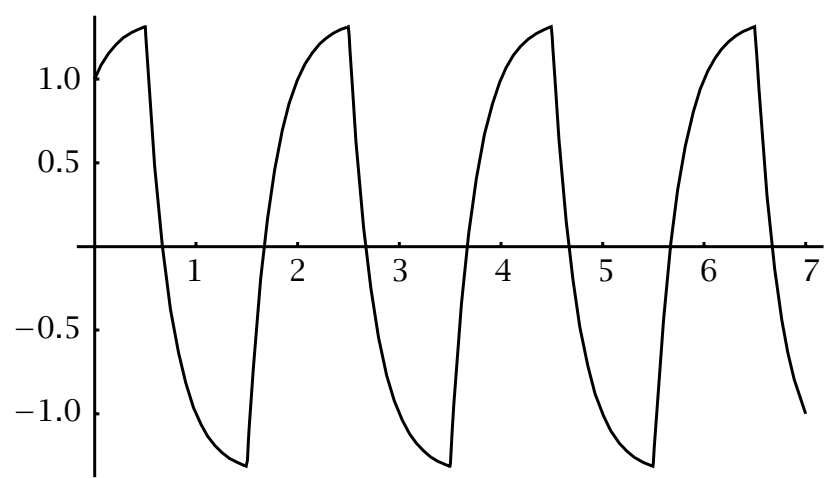

FIGURE 3.5. $T_{j}(t)$ for $b(t)=b, b=\cosh [2], j=2, a=1 / \pi$.

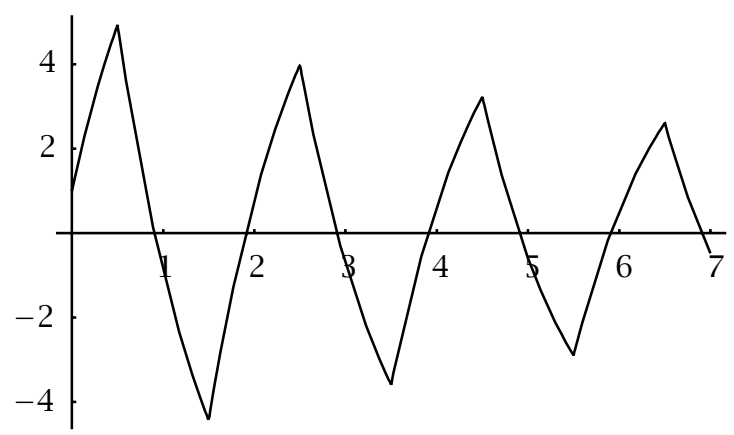

FIGURE 3.6. $T_{j}(t)$ for $b(t)=b, b=1.1, j=1, a=1 / \pi$.

If $j>R$, as is the case for all large $j$, the ratio satisfies $-1<r(j)<0$. So our solutions $T_{j}$ oscillate and dampen with time to zero. See Figure 3.6.

Finally, if $j=Q$, then $T_{j}(t)=0$ for all $t \geq 0$ for certain initial condition $u_{0}(x)$. To 
see this, realize that this condition is equivalent to

$$
b-e^{d_{j} / 2}=0,
$$

and so we cannot use equation (3.11) for $T_{j}(t)$, but equation (2.33) does apply and becomes, in the scalar case,

$$
T_{j}(t)=\frac{b-e^{-d_{j}(t-n)}}{b-1} T_{j}(n) .
$$

Because we require $T_{j}(t)$ to be continuous,

$$
T_{(n+1) j}\left(n+\frac{1}{2}\right)=\lim _{t \rightarrow(n+1 / 2)^{-}} T_{n j}(t) \equiv T_{n j}\left(n+\frac{1}{2}\right),
$$

where $T_{n j}$ denotes the restriction of the solution $T_{j}$ to the interval $n-1 / 2 \leq t<$ $n+1 / 2$. Therefore, by our assumption $b>1$ and equation (3.21),

$$
T_{(n+1) j}\left(n+\frac{1}{2}\right)=\frac{b-e^{d_{j} / 2}}{b-1} T_{(n+1) j}(n+1)=0,
$$

and so

$$
0=T_{n j}\left(n+\frac{1}{2}\right)=\frac{b-e^{-d_{j} / 2}}{b-1} T_{n j}(n) .
$$

Since

$$
b-e^{-d_{j} / 2} \neq 0
$$

for $b>1, T_{n j}(n)=0$. Hence, by equation (3.21), $T_{n j}(t)=0$ for all $t$ in the interval $n-1 / 2 \leq t<n+1 / 2$. As this argument applies for any $n$, we have $T_{j}(t)=0$ for all $t \geq 0$.

Since $T_{j}(0)=0$ and since $T_{j}(0)$ is the Fourier coefficient of the initial condition by equation (3.14), we conclude that $u_{0}(x)$ must be orthogonal to $X_{j}(x)=\sqrt{2} \sin (\pi j x)$. Otherwise, the superposition series of equation (3.12) cannot represent $u_{0}(x)$ as we do not have equality when $t=0$. The separation of variables method fails to yield a solution if the initial condition $u_{0}(x)$ is not orthogonal to $\sin (\pi j x)$ for this value of $j$.

The nondelay equation (3.26), described below, has solutions $T_{j}$ (3.27) which increase monotonically and without bound for all $j$ if $b>1$ and $T_{j}(0) \neq 0$.

REMARK. For comparison, the nondelay equation

$$
u_{t}(x, t)=a^{2} u_{x x}(x, t)+b u_{t}(x, t)
$$

has, upon application of separation of variables, solution functions

$$
T_{j}(t)=e^{d_{j} t /(b-1)} T_{j}(0) .
$$

Since $d_{j}>0$ for all $j=1,2,3, \ldots$, the $T_{j}(t)$ functions are either monotonically increasing without bound or monotonically decaying to zero, depending on whether $b>1$ 
or $b<1$. Therefore, the appearance of oscillations in the EPCA problem (3.1) is a fundamental difference of this type of problem from problems without delay. This phenomenon has been observed before in [1, 2]. However, what is unique about the work of this paper is the appearance of periodic solutions. As we will see in the following examples, some of the periodic solutions involve oscillation (crossing of the time axis for arbitrarily large $t$ ), while others do not cross the axis at all. Also, the period of the periodic solutions become an issue as we exhibit a problem which has several periodic solutions with periods different from the piecewise constant argument $t-[t+1 / 2]$, which has period 1 .

REMARK. For $b=1$, equation (3.9) is not defined and our assumption in Theorem 2.2 that $B-I=b-1$ be nonsingular does not hold. In fact, our problem (3.1) does not have a nonzero solution that is a superposition of solutions of the form described above. The same is true of the nondelay equation as the reader can verify for himself. To see this for the delay equation, let $b=1$ and substitute $n$ for $t$ in equation (3.6) to obtain

$$
T_{j}^{\prime}(n)=-d_{j} T_{j}(n)+T_{j}^{\prime}(n), \quad j=1,2,3, \ldots
$$

or

$$
T_{n j}(n)=0, \quad n=0,1,2, \ldots, \quad j=1,2,3, \ldots .
$$

In particular, $T_{j}(0)=T_{0 j}(0)=0$ for all $j=1,2,3, \ldots$. Hence, when we substitute $t=0$ into the superposition equation (3.12) of the solutions $X_{j}(x) T_{j}(t)$, we have

$$
u(x, 0)=u_{0}(x)=\sum_{j=1}^{\infty} X_{j}(x) T_{j}(0)=0
$$

and we see that the separation of variables does not provide a solution for $u_{0}(x) \neq 0$, but does yield the trivial solution for $u_{0}(x) \equiv 0$.

Note that the nondelay equation (3.26) has no solution for $u_{0}(x) \neq 0$ and only the trivial solution for $u_{0}(x)=0$.

4. Generalizations to scalar time-dependent $B=b(t)$. We can generalize our problem (2.1), (2.2), and (2.3) in the scalar case by replacing the constant matrix $B$ with scalar function $b(t)$ to obtain

$$
u_{t}(x, t)=a^{2} u_{x x}(x, t)+b(t) u_{t}\left(x,\left[t+\frac{1}{2}\right]\right),
$$

where we impose the same homogeneous boundary conditions

$$
u(0, t)=0, \quad u(1, t)=0,
$$

and the same initial condition

$$
u(x, 0)=u_{0}(x)
$$

Solving this by using separation of variables, as before, produces the following versions of the corresponding equations from Section 2

$$
\frac{T^{\prime}(t)-b(t) T^{\prime}\left(\left[t+\frac{1}{2}\right]\right)}{a^{2} T(t)}=\frac{X^{\prime \prime}(x)}{X(x)}=-P^{2} .
$$


As in Section 2, the boundary conditions require that $P$ be any of the numbers $\lambda_{j}=\pi j$, $j=1,2,3, \ldots$, and that for $P=\lambda_{j}$

$$
X_{j}(x)=\sqrt{2} \sin (\pi j x) \quad j=1,2,3, \ldots .
$$

Also, for $P=\lambda_{j}$ the time equation becomes

$$
T_{j}^{\prime}(t)=-a^{2} \lambda_{j}^{2} T_{j}(t)+b(t) T_{j}^{\prime}\left(\left[t+\frac{1}{2}\right]\right) .
$$

This can be easily solved on the individual time intervals $n-0.5 \leq t<n+0.5$ because $[t+1 / 2]=n$, a constant, on these intervals. If we denote by $T_{n j}(t)$ the solution of this equation on the interval $n-0.5 \leq t<n+0.5$ and if we let $d_{j}=a^{2} \lambda_{j}^{2}$, then

$$
T_{n j}(t)=C_{n j} e^{-d_{j}(t-n)}+B_{n j} e^{-d_{j} t} \int b(t) e^{d_{j} t} d t,
$$

where the integral on the right-hand side represents any antiderivative of $b(t)$ and where $B_{n j}$ is a constant that is defined by

$$
B_{n j} \equiv T_{n j}^{\prime}\left(\left[n+\frac{1}{2}\right]\right)=T_{n j}^{\prime}(n) .
$$

To find a more useful formula for $B_{n j}$, we let $t=n$ in (4.6) to obtain

$$
B_{n j}=\frac{d_{j} T_{n j}(n)}{b(n)-1},
$$

provided $b(n) \neq 1$ for all $n=0,1,2,3, \ldots$. Furthermore, if we define the function

$$
E_{n j}(t) \equiv \frac{d_{j} e^{-d_{j} t}}{b(n)-1} \int b(t) e^{d_{j} t} d t
$$

we can write our time solutions on $[n-0.5, n+0.5)$ as

$$
T_{n j}(t)=C_{n j} e^{-d_{j}(t-n)}+E_{n j}(t) T_{n j}(n)
$$

for $b(n) \neq 1$ and $n=0,1,2,3, \ldots$.

To find a formula for $C_{n j}$, we let $t=n$ in (4.11) to obtain

$$
C_{n j}=\left[1-E_{n j}(n)\right] T_{n j}(n) .
$$

If we define the constant

$$
D_{n j}=1-E_{n j}(n), \quad n=0,1,2,3, \ldots,
$$

we can write

$$
T_{n j}(t)=\left[D_{n j} e^{-d_{j}(t-n)}+E_{n j}(t)\right] T_{n j}(n),
$$

provided $b(n) \neq 1$ for $n=0,1,2,3, \ldots$.

In order to preserve continuity, we must have the solutions $T_{n j}(t)$ and $T_{(n-1) j}(t)$ to be equal at $t=n-0.5$. Therefore,

$$
T_{n j}(n-0.5)=\left[D_{n j} e^{d_{j} / 2}+E_{n j}(n-0.5)\right] T_{n j}(n)
$$


must equal

$$
\lim _{t \rightarrow(n-0.5)^{-}} T_{(n-1) j}=\left[D_{(n-1) j} e^{-d_{j} / 2}+E_{(n-1) j}(n-0.5)\right] T_{(n-1) j}(n-1)
$$

yielding

$$
T_{n j}(n)=F_{j}(n) T_{(n-1) j}(n-1),
$$

where we have defined the function

$$
F_{j}(n) \equiv \frac{D_{(n-1) j} e^{-d_{j} / 2}+E_{(n-1) j}(n-0.5)}{D_{n j} e^{d_{j} / 2}+E_{n j}(n-0.5)},
$$

provided

$$
D_{n j} e^{d_{j} / 2}+E_{n j}(n-0.5) \neq 0 \text { for all } n=0,1,2,3, \ldots .
$$

This relation allows us to relate $T_{n j}(n)$ to $T_{j}(0)=T_{0 j}(0)$ by the following calculations

$$
\begin{aligned}
T_{n j}(n) & =F_{j}(n) T_{(n-1) j}(n-1) \\
& =F_{j}(n) F_{j}(n-1) T_{(n-2) j}(n-2) \\
& =F_{j}(n) F_{j}(n-1) F_{j}(n-2) T_{(n-3) j}(n-3) \\
& =\cdots \\
& =F_{j}(n) F_{j}(n-1) F_{j}(n-2) \cdots F_{j}(2) F_{j}(1) T_{0 j}(0) \\
& =\left[\prod_{k=1}^{n} F_{j}(k)\right] T_{j}(0) .
\end{aligned}
$$

Therefore, we can write the solution $T_{n j}(t)$ on the interval $n-0.5 \leq t<n-0.5$ as

$$
T_{n j}(t)=\left[D_{n j} e^{-d_{j}(t-n)}+E_{n j}(t)\right]\left[\prod_{k=1}^{n} F_{j}(k)\right] T_{j}(0),
$$

where $d_{j}=a^{2} \lambda_{j}^{2}, n=[t+1 / 2]$,

$$
\begin{aligned}
E_{n j}(t) & \equiv \frac{d_{j} e^{-d_{j} t}}{b(n)-1} \int b(t) e^{d_{j} t} d t, \\
D_{n j} & \equiv 1-E_{n j}(n), \\
F_{j}(n) & \equiv \frac{D_{(n-1) j} e^{-d_{j} / 2}+E_{(n-1) j}(n-0.5)}{D_{n j} e^{d_{j} / 2}+E_{n j}(n-0.5)},
\end{aligned}
$$

and where we define

$$
\prod_{k=1}^{0} F_{j}(k) \equiv 1 .
$$

We also require that $b(n) \neq 1$ for all $n=0,1,2,3, \ldots$ and that

$$
D_{n j} e^{d_{j} / 2}+E_{n j}(n-0.5) \neq 0 \text { for all } n=1,2,3,4 \ldots .
$$

We complete our discussion of the solution to problem (4.1) by summarizing our calculations in the following theorem. 
THEOREM 4.1. The solution to BVP (4.1) can be written as

$$
u(x, t)=\sum_{j=1}^{\infty} X_{j}(x) T_{j}(t),
$$

where $X_{j}$ is given by

$$
X_{j}(x)=\sqrt{2} \sin (\pi j x), \quad j=1,2,3, \ldots
$$

and $T_{j}$ is given by

$$
T_{j}(t)=\left[D_{n j} e^{-d_{j}(t-n)}+E_{n j}(t)\right]\left[\prod_{k=1}^{n} F_{j}(k)\right] T_{j}(0),
$$

with $d_{j}=a^{2} \pi^{2} j^{2}$ and $n=[t+1 / 2]$. The functions $E_{n j}, D_{n j}$, and $F_{j}$ are given in (4.22), (4.23), (4.24), (4.25), and (4.26). Also, the initial value $T_{j}(0)$ is determined, as in Section 3, by

$$
T_{j}(0)=\hat{u}_{0}(j)=\sqrt{2} \int_{0}^{1} u_{0}(x) \sin (\pi j x) d x
$$

REMARK. The reader can verify for himself that formula (4.29) reduces to (3.11) for $b(t)=b$.

REMARK. In the previous discussion, we required that condition (4.26) holds for all $n=1,2,3,4, \ldots$. We show that should this condition be violated, the Fourier method does not provide solutions except for restricted conditions on $u_{0}(x)$. To see this, suppose

$$
D_{n j} e^{d_{j} / 2}+E_{n j}(n-0.5)=0
$$

for $n=k$, but that

$$
D_{n j} e^{-d_{j} / 2}+E_{n j}(n+0.5) \neq 0
$$

for all $n=0,1,2,3, \ldots, k-1$. If we let $t=k-0.5$ in (4.14), we get

$$
T_{k j}(k-0.5)=\left[D_{k j} e^{d_{j} / 2}+E_{k j}(k-0.5)\right] T_{k j}(k)=0 .
$$

Since the solutions $T_{k j}$ and $T_{(k-1) j}$ must be equal at $k-0.5$, then

$$
\begin{aligned}
0 & =T_{k j}(k-0.5) \\
& =\lim _{t \rightarrow(k-0.5)^{-}} T_{(k-1) j}(t) \\
& =\left[D_{(k-1) j} e^{-d_{j} / 2}+E_{(k-1) j}(k-0.5)\right] T_{(k-1) j}(k-1),
\end{aligned}
$$

so that by our assumptions $T_{(k-1) j}(k-1)=0$. Therefore, by (4.14), for all $t \in[k-$ $1.5, k-0.5]$,

$$
T_{(k-1) j}(t)=\left[D_{(k-1) j} e^{-d_{j}(t-k+1)}+E_{(k-1) j}(t)\right] T_{(k-1) j}(k-1)=0 .
$$


In particular, $T_{(k-1) j}(k-1.5)=0$. Now, if we match our solutions at $t=k-1.5$, we get

$$
\begin{aligned}
0 & =T_{(k-1) j}(k-1.5) \\
& =\lim _{t \rightarrow(k-1.5)^{-}} T_{(k-2) j}(t) \\
& =\left[D_{(k-2) j} e^{-d_{j} / 2}+E_{(k-2) j}(k-1.5)\right] T_{(k-2) j}(k-2),
\end{aligned}
$$

and so $T_{(k-2) j}(k-2)=0$ and also $T_{(k-2) j}(t)=0$ for all $t \in[k-2.5, k-1.5]$ by (4.14). Continuing in this fashion, we have $T_{j}(t)=0$ on the interval $[0, k-0.5]$ and, in particular, $T_{j}(0)=0$. So, by (4.29), $T_{j}(t)=0$ for all $t \geq 0$. Also, by letting $t=0$ in (4.27), we get

$$
u_{0}(x)=\sum_{i \neq j} T_{i}(0) X_{i}(x) .
$$

If $u_{0}(x)$ cannot be completely expressed by this series, i.e., if $\hat{u}_{0}(j) \neq 0$, then the Fourier method described in this paper does not provide the solution since $\hat{u}_{0}(j)=$ $T_{j}(0)$.

For the example of $b(t)=b$, we saw the consequences of the violation of condition (4.26). Indeed, this condition does not hold for $b>1$ when $j$ satisfies

$$
-e^{d_{j} / 2}+b=0
$$

i.e., when

$$
j=\sqrt{\frac{2 \ln b}{a^{2} \pi^{2}}},
$$

(see Section 3). As noted in Section 3 and above, we must have $\hat{u}_{0}(j)=0$ in order for our method to describe the solution. More importantly, the value of $j$ above represents a sharp division between $T_{j}$ solutions with monotonic growth (for $j$ smaller), and $T_{j}$ solutions with growth and oscillation (for $j$ larger). For $j$ assuming this critical value, $T_{j}(t) \equiv 0$.

5. A scalar EPCA for $b(t)=b \cos (2 \pi t)$. As we seek periodic solutions, a natural time-dependent function $b(t)$ to consider is $b(t)=b \cos (\alpha t)$, which has period $2 \pi / \alpha$, for some real number $\alpha$. In this section, we discuss in detail $\cos (2 \pi t)$, which has the same period as does the expression $t-n=t-[t+1 / 2]$ found in (4.29). We do not discuss the cases when $\alpha$ is a multiple of $2 \pi$ as they generate similar solution behavior. However, in the next section we discuss $\alpha=\pi$ which exhibits very different solution properties.

To begin, we first write explicitly the solution (4.29) to the BVP (4.1) with $b(t)=$ $b \cos (\alpha t)$ for any $\alpha$. We need the indefinite integral

$$
\int b(t) e^{d_{j} t} d t=\frac{b e^{d_{j} t}}{\sqrt{d_{j}^{2}+\alpha^{2}}} \cos (\alpha t-\gamma),
$$

where $\gamma$ is defined by

$$
\cos \gamma=\frac{d_{j}}{\sqrt{d_{j}^{2}+\alpha^{2}}}, \quad \sin \gamma=\frac{\alpha}{\sqrt{d_{j}^{2}+\alpha^{2}}}
$$


to yield, by (4.22),

$$
E_{n j}(t)=\frac{b d_{j}}{\sqrt{d_{j}^{2}+\alpha^{2}}} \frac{\cos (\alpha t-\gamma)}{b \cos (\alpha n)-1},
$$

which for $\alpha=2 \pi$ becomes

$$
E_{n j}(t)=\frac{b d_{j}^{2}}{(b-1)\left(d_{j}^{2}+\alpha^{2}\right)}\left[\cos (\alpha t)+\frac{\alpha}{d_{j}} \sin (\alpha t)\right],
$$

provided $b \neq 1$. In what follows it is convenient to define the parameter

$$
p_{j}=\frac{d_{j}^{2}+\alpha^{2}(1-b)}{b d_{j}^{2}} \text {. }
$$

This allows us to write

$$
E_{n j}(t)=\frac{-1}{p_{j}-1}\left[\cos (\alpha t)+\frac{\alpha}{d_{j}} \sin (\alpha t)\right]
$$

and since $D_{n j}=1-E_{n j}(n)$, by (4.23), we have

$$
D_{n j}=\frac{p_{j}}{p_{j}-1} .
$$

Hence,

$$
\begin{aligned}
H_{j}(t) & \equiv\left[D_{n j} e^{-d_{j}(t-n)}+E_{n j}(t)\right] \\
& =\frac{1}{p_{j}-1}\left[p_{j} e^{-d_{j}(t-n)}-\cos (\alpha t)-\frac{\alpha}{d_{j}} \sin (\alpha t)\right] .
\end{aligned}
$$

The ratio function $F_{j}(n)$, given by (4.24), in this case, turns out to be independent of $n$ since

$$
F_{j}(n)=\frac{p_{j} e^{-d_{j} / 2}+1}{p_{j} e^{d_{j} / 2}+1} .
$$

Therefore, by (4.29),

$$
\begin{aligned}
T_{j}(t) & =\frac{1}{p_{j}-1}\left[p_{j} e^{-d_{j}(t-n)}-\cos (\alpha t)-\frac{\alpha}{d_{j}} \sin (\alpha t)\right]\left(\frac{p_{j} e^{-d_{j} / 2}+1}{p_{j} e^{d_{j} / 2}+1}\right)^{n} T_{j}(0) \\
& =H_{j}(t)\left(\frac{p_{j} e^{-d_{j} / 2}+1}{p_{j} e^{d_{j} / 2}+1}\right)^{n} T_{j}(0) .
\end{aligned}
$$

Since $\alpha=2 \pi$ and $n=[t+1 / 2], H_{j}(t)$ is 1-periodic and so the behavior of the solutions $T_{j}(t)$ is primarily governed by the ratio

$$
r(j)=F_{j}(n)=\frac{p_{j} e^{-d_{j} / 2}+1}{p_{j} e^{d_{j} / 2}+1},
$$

though we shall see that the behavior of $H_{j}(t)$ will also play a role, something that was not the case for $b(t)=b$.

To analyze the behavior of the solutions $T_{j}(t)$, we make use of the parameters $d_{j}$ and $p_{j}$ to classify the solutions. This is possible because only $d_{j}$ and $p_{j}$ appear in the ratio $r(j)$ above. This is also necessary as it is not possible in all cases to solve for $j$ explicitly in terms of $a$ and $b$ as was done for $b(t)=b$. To determine how $T_{j}(t)$ 
behaves, use $j, a$, and $b$ to compute $d_{j}$ and $p_{j}$, and then determine which relationship below $d_{j}$ and $p_{j}$ satisfy. The behavior of $T_{j}$ is described in the text accompanying the relation.

THEOREM 5.1. The solution to $B V P$ (4.1) with $b(t)=b \cos (2 \pi t), b \neq 0,1$, is as described in Theorem 4.1 with $T_{j}(t)$ given by (5.10). The time functions $T_{j}(t)$ behave as follows:

(a) For $p_{j}<-e^{d_{j} / 2}, T_{j}(t)$ dampens to zero and may or may not oscillate.

(b) For $p_{j}=-e^{d_{j} / 2}, T_{j}(t)=0$ for all $t \geq 1 / 2$.

(c) For $-e^{d_{j} / 2}<p_{j}<-1 / \cosh \left(d_{j} / 2\right), T_{j}(t)$ oscillates and dampens to zero as $t \rightarrow \infty$.

(d) For $p_{j}=-1 / \cosh \left(d_{j} / 2\right), T_{j}(t)$ is an oscillating, 2-periodic solution.

(e) For $-1 / \cosh \left(d_{j} / 2\right)<p_{j}<-e^{-d_{j} / 2}, T_{j}(t)$ is oscillating and unbounded as $t \rightarrow \infty$.

(f) For $p_{j}=-e^{-d_{j} / 2}, T_{j}(t) \equiv 0$ for all $t \geq 0$ if $u_{0}(x)$ is orthogonal to $\sqrt{2} \sin (\pi j x)$; otherwise separation of variables does not provide a solution.

(g) For $-e^{-d_{j} / 2}<p_{j}<0, T_{j}(t)$ is oscillating and unbounded as $t \rightarrow \infty$.

(h) For $p_{j}=0, T_{j}(t)$ is an oscillating, 1-periodic solution.

(i) For $0<p_{j}<1, T_{j}(t) \rightarrow 0$ as $t \rightarrow \infty$ and oscillates.

(j) For $p_{j}=1$, separation of variables does not provide a solution.

(k) For $p_{j}>1, T_{j}(t) \rightarrow 0$ as $t \rightarrow \infty$ and may or may not oscillate.

Proof. The cases listed in the theorem can most readily be discerned by examining the graph of the ratio function $r(j)$, not as a function of $j$, but as a function of $p_{j}$. This is done in Figure 5.1. Again, if $|r(j)|>1$, then $T_{j}(t)$ grows without bound while if $|r(j)|<1$, then $T_{j}(t)$ dampens to zero. If $|r(j)|=1$, the solutions are periodic. If $r(j)<0$, then we are guaranteed to have oscillations while if $r(j)>0$, then we may or may not have oscillations depending on the behavior of $H_{j}(t)$. These remarks are

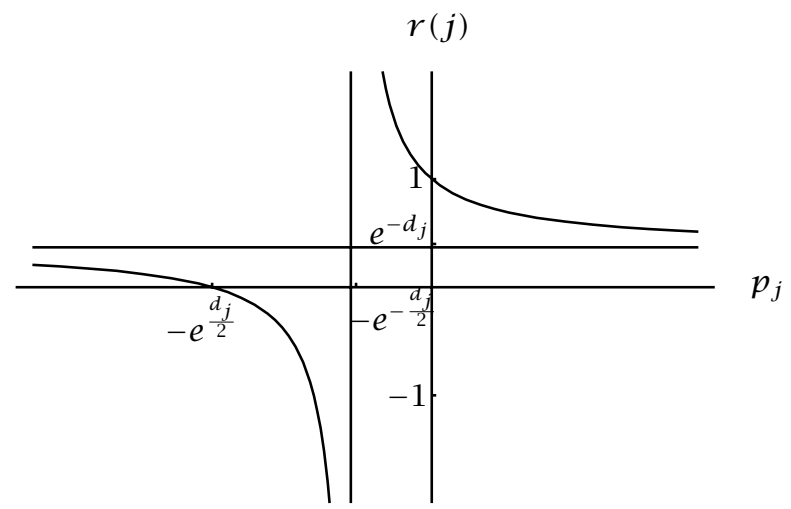

FIGURE 5.1. Ratio function $r(j)$ for $b(t)=b \cos (2 \pi t), j=1, a=1 / \pi$.

sufficient to completely prove (c), (d), and (e) of the theorem as $r(j)$ is negative for these values of $p_{j}$. See Figure 5.1. In fact, the ratio $r(j)=-1$ in case (d) guaranteeing that $H_{j}(t)$ must cross the time axis an odd number of times in each interval [ $n-$ $1 / 2, n+1 / 2)$. To see this, recall that in order to ensure continuity of $T_{j}$ at the endpoints of these intervals, the ratio $r(j)$ must act in a way so as to join together the segments 
of $H_{j}(t)$ in adjacent intervals of the form $[n-1 / 2, n+1 / 2)$. Since the ratio is negative, the values of $H_{j}(t)$ near the endpoints of each of these intervals must be of opposite sign and, consequently, $H_{j}(t)$ must cross the axis an odd number of times. Hence, $T_{j}(t)$ must be 2-periodic, not 1-periodic, as $H_{j}(t)$ requires two unit intervals to repeat.

Also, from Figure 5.1, the ratio $r(j)=0$ for $p_{j}=-e^{d_{j} / 2}$ so that $T_{j}(t) \equiv 0$ for all $t \geq 1 / 2$, as can be seen from (5.10). On $[0,1 / 2), T_{j}(t)=H_{j}(t) T_{j}(0)$. This gives (b).

In case (f) the ratio $r(j)$ is not defined since condition (4.26) is violated, i.e.,

$$
D_{n j} e^{d_{j} / 2}+E_{n j}(n-0.5)=\frac{p_{j} e^{d_{j} / 2}+1}{p_{j}-1}=0,
$$

and so by the second remark following Theorem 4 , we see that $T_{j}(t) \equiv 0$ for $t \geq 0$ if $u_{0}(x)$ is orthogonal to $\sin (\pi j x)$. If $u_{0}$ is not orthogonal to $\sqrt{2} \sin (\pi j x)$, the Fourier method does not provide a solution.

In the remaining six cases, (a) and (g) through (k), the ratio is positive and so we must examine the function $H_{j}$ to see if there are oscillations. For the problem $b(t)=b$, the function (3.11),

$$
\left(\frac{b-e^{-d_{j}(t-[t+1 / 2])}}{b-1}\right)
$$

is always increasing or decreasing on $[n-1 / 2, n+1 / 2)$ so that if the corresponding $T_{j}$ function oscillates, it is because this function has crossed the axis once and once only. The ratio function in that case must be negative to ensure continuity. However, for $b(t)=b \cos (2 \pi t)$, the function $H_{j}(t)$ may cross the axis 0,1 , or 2 times on a single unit interval $[n-1 / 2, n+1 / 2)$. If it crosses twice, the ratio function must be positive for the solutions to match up at $n+1 / 2, n=0,1,2, \ldots$. Therefore, we must look closely at the behavior of $H_{j}$ to determine if there are oscillations.

To illustrate, consider case (a), where $0<r(j)<e^{-d_{j}}<1$ so that the solutions $T_{j}(t)$ dampen to zero. Whether the $T_{j}(t)$ solutions oscillate depends on $d_{j}$ and $p_{j}$. For all values of $d_{j}$ and $p_{j}$, we have

$$
\begin{aligned}
H_{j}\left(n-\frac{1}{2}\right) & =\frac{p_{j} e^{d_{j} / 2}+1}{p_{j}-1}, \quad H_{j}(n)=1, \\
\lim _{t \rightarrow(n+1 / 2)^{-}} H_{j}(t) & =\frac{p_{j} e^{-d_{j} / 2}+1}{p_{j}-1} .
\end{aligned}
$$

For $p_{j}<-e^{d_{j} / 2}$, these are all positive. However, for $t=n-1 / 4$,

$$
H_{j}\left(n-\frac{1}{4}\right)=\frac{1}{p_{j}-1}\left[p_{j} e^{d_{j} / 4}+\frac{\alpha}{d_{j}}\right],
$$

so $H_{j}(n-1 / 4)<0$ for small $d_{j}>0$ and, therefore, $T_{j}(t)$ oscillate and dampen to zero. On the other hand, $H_{j}(t)>0$ for large $d_{j}$ and all $t$ so that $T_{j}(t)$ go to zero without oscillation.

For case $(\mathrm{g}), r(j)>1$ and, hence, $T_{j}(t)$ is unbounded. In this case, we note by (5.14) that $H_{j}(n-1 / 2)$ and $\lim _{t \rightarrow(n+1 / 2)-} H_{j}(t)$ are negative, but that $H_{j}(n)=1$ so that $T_{j}(t)$ 
crosses the axis twice on the interval $[n-1 / 2, n+1 / 2)$. Therefore, $T_{j}(t)$ has growing oscillations.

For case (h), the ratio $r(j)=1$ so that $T_{j}(t)=H_{j}(t) T_{j}(0)$, i.e., $T_{j}(t)$ is a 1-periodic function. This function crosses the axis twice on each interval $[n-1 / 2, n+1 / 2)$ since $H_{j}(n-1 / 2)=-1, H_{j}(n)=1$, and $\lim _{t \rightarrow(n+1 / 2)^{-}} H_{j}(t)=-1$. Hence, $T_{j}(t)$ is an oscillating, periodic solution.

Cases (i) and (k) can be handled by noting that, regardless of the value of $b$, if $p_{j}>0$ and $p_{j} \neq 1$, then the ratio $r(j)$ satisfies $0<e^{-d_{j}}<r(j)<1$ so that the solutions dampen to zero as $t \rightarrow+\infty$. To see whether they go to zero with or without oscillation, we must consider $H_{j}(t)$. For $0<p_{j}<1$, formulas (5.14) give $H_{j}(n-1 / 2)<0, H_{j}(n)=1$ and $\lim _{t \rightarrow(n+1 / 2)^{-}} H_{j}(t)<0$ so that $H_{j}(t)$ must cross the axis twice on each interval $[n-1 / 2, n+1 / 2)$. Hence, $T_{j}(t)$ must oscillate as well as dampen to zero.

For $p_{j}>1, T_{j}(t)$ may or may not oscillate. Generally, if $p_{j}$ is large, $T_{j}(t)$ goes to zero without oscillation. For example, if $p_{j}=20$ and $d_{j}=10$, then $b=0.068, H_{j}(t)$ is positive on $[n-1 / 2, n+1 / 2)$, and, hence, $T_{j}(t)$ goes to zero without oscillation. If, on the other hand, $p_{j}>1$, but not too much bigger than 1 , then $H_{j}(t)$ crosses the axis twice so that $T_{j}(t)$ oscillates as it approaches zero. For example, if $b=0.9$ and $d_{j}=2 \pi / 1.5$ (chosen by adjusting $a$ in $d_{j}=(a \pi j)^{2}$ ), then $p_{j}=1.36, H_{j}(n)=1$, and $H_{j}(n+1 / 4)=-2.83$, so that $H_{j}(t)$ must cross the axis on the interval $[n, n+1 / 4]$. $H_{j}(t)$ also crosses the axis on $[n+1 / 4, n+1.4)$ since $H_{j}(1.4)=0.5$. Hence, $T_{j}(t)$ must oscillate and dampen to zero as $t \rightarrow+\infty$.

For the last case (j) note that $p_{j}=1$ if and only if $b=1$. If $b=1$, then the functions $E_{n j}(t)$ are not defined. In fact, our method does not provide a solution if $p_{j}=1$ for the same reason as for the case of $b(t)=b$ with $b=1$. To recapitulate, note that if we let $t=n$ in (4.6), we obtain $T_{j}(n)=0$ for all $n$ and all $j$. In particular, $T_{j}(0)=0$ for all $j=1,2,3, \ldots$ so that if we let $t=0$ in the superposition series (4.27), we get $u_{0}(x)=u(x, 0)=0$ and so our method does not provide a solution for $u_{0}(x) \neq 0$.

Finally, note that for any values of $d_{j}$ and $p_{j}$ which satisfy $p_{j} d_{j}^{2}+(2 \pi)^{2} \neq 0$, we can find a corresponding value for $b$ determined by

$$
b=\frac{d_{j}^{2}+(2 \pi)^{2}}{p_{j} d_{j}^{2}+(2 \pi)^{2}} .
$$

(This follows from the definition of $p_{j}$.) Therefore, in each of the cases listed above for which a solution exists, we can find a value of $b$ which generates a solution $T_{j}(t)$ that behaves as that case describes.

REMARK. Regardless of the values of the parameters $a$ and $b$, as $j \rightarrow+\infty$ note that $d_{j}=(a \pi j)^{2} \rightarrow+\infty$ and that

$$
p_{j}=\frac{1}{b}+\frac{(2 \pi)^{2}}{d_{j}^{2}}\left(1-\frac{1}{b}\right) \rightarrow \frac{1}{b} .
$$

So

$$
\lim _{j \rightarrow+\infty} r(j)=\lim _{j \rightarrow+\infty} \frac{p_{j} e^{-d_{j} / 2}+1}{p_{j} e^{d_{j} / 2}+1}=0 .
$$


Hence, for large $j, T_{j}(t)$ dampens to zero. Furthermore, this decay is fast as the ratio goes to zero rapidly as $j \rightarrow+\infty$ because of the exponential terms in the definition of the ratio $r(j)$. Consequently, for large $t$ the series in (4.27) for $u(x, t)$ has only a small number of terms that are not approximately zero.

6. A scalar EPCA for $b(t)=b \cos (\pi t)$. We continue the discussion of the previous section by investigating a time dependent function $b(t)$ having a period different from that of the expression $t-n=t-[t+1 / 2]$, which has period 1 and appears in (4.29). In particular, we consider $b(t)=b \cos (\pi t)$, which has period 2 , and which yields periodic solutions $T_{j}$ of two different periods, as well as producing nonperiodic solutions.

We begin by writing the analytic expressions for $T_{j}$. Into (5.3), we substitute $\alpha=\pi$ to obtain

$$
E_{n j}(t)=\frac{b d_{j}^{2}}{b \cos (\pi n)-1}\left[\frac{1}{d_{j}^{2}+\pi^{2}}\right]\left[\cos (\pi t)+\frac{\pi}{d_{j}} \sin (\pi t)\right],
$$

provided $b \neq-1,1$. By (4.22), (4.23), and (4.24), we have

$$
\begin{gathered}
E_{n j}(n-0.5)= \begin{cases}\frac{-b d_{j} \pi}{\left(d_{j}^{2}+\pi^{2}\right)(1+b)}, & \text { if } n \text { odd, } \\
\frac{b d_{j} \pi}{\left(d_{j}^{2}+\pi^{2}\right)(1-b)}, & \text { if } n \text { even, }\end{cases} \\
E_{(n-1) j}(n-0.5)= \begin{cases}\frac{-b d_{j} \pi}{\left(d_{j}^{2}+\pi^{2}\right)(1-b)}, & \text { if } n \text { odd, } \\
\frac{b d_{j} \pi}{\left(d_{j}^{2}+\pi^{2}\right)(1+b)}, & \text { if } n \text { even, }\end{cases}
\end{gathered}
$$

and

$$
D_{n j}= \begin{cases}1-\frac{b d_{j}^{2}}{\left(d_{j}^{2}+\pi^{2}\right)(1+b)}, & \text { if } n \text { odd, } \\ 1+\frac{b d_{j}^{2}}{\left(d_{j}^{2}+\pi^{2}\right)(1-b)}, & \text { if } n \text { even. }\end{cases}
$$

Combining these, we see that the ratio $F_{j}(n)$, unlike the previous examples, does indeed depend on $n$, though it depends only on whether $n$ is odd or even. To be specific

$$
F_{j}(n)= \begin{cases}\left(\frac{1+b}{1-b}\right)\left[\frac{\left[d_{j}^{2}+\pi^{2}(1-b)\right] e^{-d_{j} / 2}-b d_{j} \pi}{\left[d_{j}^{2}+\pi^{2}(1+b)\right] e^{d_{j} / 2}-b d_{j} \pi}\right], & \text { if } n \text { odd, } \\ \left(\frac{1-b}{1+b}\right)\left[\frac{\left[d_{j}^{2}+\pi^{2}(1+b)\right] e^{-d_{j} / 2}+b d_{j} \pi}{\left[d_{j}^{2}+\pi^{2}(1-b)\right] e^{d_{j} / 2}+b d_{j} \pi}\right], & \text { if } n \text { even. }\end{cases}
$$

By (4.21), we have

$$
T_{j}(t)=H_{j}(t)\left[\prod_{k=1}^{n} F_{j}(k)\right] T_{j}(0)
$$


where $n=[t+1 / 2]$ and where $H_{j}(t)=D_{n j} e^{-d_{j}(t-n)}+E_{n j}(t)$ is given by

$$
H_{j}(t)= \begin{cases}\frac{\left[\left[d_{j}^{2}+\pi^{2}(1+b)\right] e^{-d_{j}(t-n)}-b d_{j}^{2} \cos (\pi t)-b d_{j} \pi \sin (\pi t)\right]}{\left(d_{j}^{2}+\pi^{2}\right)(1+b)}, & \text { if } n \text { odd, } \\ \frac{\left[\left[d_{j}^{2}+\pi^{2}(1-b)\right] e^{-d_{j}(t-n)}-b d_{j}^{2} \cos (\pi t)-b d_{j} \pi \sin (\pi t)\right]}{\left(d_{j}^{2}+\pi^{2}\right)(1-b)}, & \text { if } n \text { even. }\end{cases}
$$

Note that $H_{j}(t)$ is periodic with period 2, not period 1 .

In the previous examples we identified the nature of the solutions $T_{j}$ by examining the ratio function $r(j)=F_{j}(n)$. If $F_{j}(n)=-1$, for example, we have oscillatory, periodic solutions. In this case, $F_{j}(n)$ varies with $n$ for $a, b$, and $j$ fixed so that if $F_{j}(n)=-1$ for some values of $n$, it might be different for other values of $n$, and, therefore, the solutions $T_{j}$ are not necessarily periodic and oscillating for all time $t$. Fortunately, though, the ratio $F_{j}(n)$ depends not on the exact value of $n$, but only on whether $n$ is odd or even. Therefore, the product of $F_{j}$ for two consecutive values of $n$, i.e., $P\left(d_{j}\right) \equiv F_{j}(n) F_{j}(n+1)$ is constant for all $n=1,2,3, \ldots$, and depends only on $j$, assuming $a$ and $b$ are fixed. Hence, if this product is -1 , then we have oscillatory, periodic solutions. As we will see, the periods of the periodic solutions are no longer 1 , but some larger value (either 2 or 4 ). In fact, since $F_{j}(n) \neq F_{j}(n+1)$ for all $n$, we have no solutions $T_{j}(t)$ of period 1 as at least one of $F_{j}(n)$ and $F_{j}(n+1)$ cannot be 1 or -1 (see (6.5)). Equivalently, $T_{j}(t)$ cannot be of period 1 as $H_{j}(t)$ is not of period 1 . We summarize and expand on these ideas in the following theorem.

THEOREM 6.1. The solution to BVP (4.1) with $b(t)=b \cos (\pi t), b \neq-1,0,1$, is as described in Theorem 4.1 with $T_{j}(t)$ given by (6.6). The time functions $T_{j}(t)$ behave as follows

(a) There are no solutions $T_{j}$ of period 1 .

(b) For $|b|<1, T_{j}(t) \rightarrow 0$ as $t \rightarrow \infty$. Furthermore, $T_{j}$ does not oscillate for small $j$, specifically, for all $j$ such that

$$
\left[d_{j}^{2}+\pi^{2}(1-|b|)\right] e^{-d_{j} / 2}-|b| d_{j} \pi
$$

is positive. For $j$ making (6.8) zero, $T(j) \equiv 0$ for all $t \geq 1 / 2$. Finally, $T_{j}$ does oscillate for all $j$ large enough such that (6.8) is negative.

(c) For $|b|>1$, there exists a unique $d_{j}^{*}>0$ satisfying

$$
\left[\left(d_{j}^{*}\right)^{2}+\pi^{2}(1-|b|)\right] e^{d_{j}^{*} / 2}+|b| d_{j}^{*} \pi=0
$$

from which follows

(1) There exists a $d_{E}>d_{j}^{*}$ such that the corresponding $T_{j}$ is a 4-periodic, oscillating solution. See Figure 6.5.

(2) For $d_{j}^{*}<d_{j}<d_{E}, T_{j}$ is unbounded and oscillating as $t \rightarrow \infty$. See Figure 6.6.

(3) For $d_{j}>d_{E}, T_{j} \rightarrow 0$ and oscillates as $t \rightarrow \infty$. See Figure 6.7.

(4) For $d_{j}<d_{j}^{*}$ and for $|b|>1$ small, $T_{j}$ is unbounded. 
(5) For $d_{j}<d_{j}^{*}$ and for $|b|>1$ large, there exists a $d_{B}<d_{j}^{*}$ such that $T_{j}$ is a 2periodic solution. See Figure 6.4. Furthermore, for $d_{j}<d_{B}, T_{j} \rightarrow 0$ as $t \rightarrow \infty$ while for $d_{B}<d_{j}<d_{j}^{*}, T_{j}$ grows without bound. See Figures 6.8 and 6.9.

REMARK. In points (4) and (5) above, we believe that the time solutions $T_{j}$ do not oscillate, but we have not been able to prove this. We need to show that $H_{j}(t)>0$ in these cases, but we have not been able to prove this although all of our computer and analytical work says that it should be so.

\section{Proof.}

Conclusion (a). This follows from the remark made immediately before the statement of the theorem.

For the other conclusions, observe that the product $P\left(d_{j}\right) \equiv F_{j}(n) F_{j}(n+1)$ becomes

$$
P\left(d_{j}\right) \equiv\left[\frac{\left[d_{j}^{2}+\pi^{2}(1-b)\right] e^{-d_{j} / 2}-b d_{j} \pi}{\left[d_{j}^{2}+\pi^{2}(1+b)\right] e^{d_{j} / 2}-b d_{j} \pi}\right]\left[\frac{\left[d_{j}^{2}+\pi^{2}(1+b)\right] e^{-d_{j} / 2}+b d_{j} \pi}{\left[d_{j}^{2}+\pi^{2}(1-b)\right] e^{d_{j} / 2}+b d_{j} \pi}\right]
$$

and is unchanged when we replace $b$ with $-b$. Because of this symmetry, we can extend our conclusions to $b<0$ by discussing the behavior of the $T_{j}$ solutions for $b>0$ only.

Conclusion (b). To prove conclusion (b) we first graph $P\left(d_{j}\right)$ as a function of $d_{j}$ (and, hence, $j$ ), and observe the essential features of the graph. We, then, prove conclusion (b) by referring to these features. Finally, we show why the graph of $P\left(d_{j}\right)$ must have the features described. For $0<b<1$, the graph of the function $P\left(d_{j}\right)$ is given in Figure 6.1.

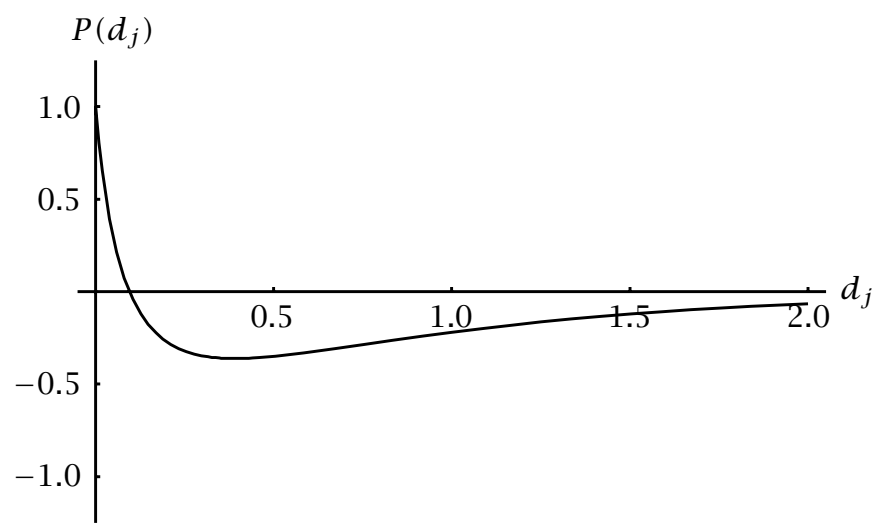

FIGURE 6.1. Product ratio $P\left(d_{j}\right)$ for $b(t)=b \cos (\pi t),|b|<1$.

The essential features of the graph that interest us are

(i) $\left|P\left(d_{j}\right)\right|<1$ for all values of $d_{j}>0$.

(ii) $P\left(d_{j}\right)$ has the same sign as (6.8) so that $P\left(d_{j}\right)$ is positive for small $d_{j}$, and negative for large $d_{j}$.

(iii) $P\left(d_{j}\right) \rightarrow 0$ as $d_{j} \rightarrow \infty$, and approaches zero from below.

As we show below, when $0<b<1$, all factors in (6.10) are positive for all $d_{j}>0$ except for expression (6.8). For large $d_{j}$ (and, hence, large $j$ ), $P\left(d_{j}\right)<0$ because (6.8) 
is also negative. This combined with (i) and (6.6) means that $T_{j}(t) \rightarrow 0$ as $t \rightarrow \infty$ and that $T_{j}$ oscillates.

When $P\left(d_{j}\right)=0(6.8)$ is also zero. Since (6.8) is in the numerator of $F_{j}(n)$ when $n$ is odd (see (6.5)), then $T_{j}(t)=0$ for all $t \geq 1 / 2$ as, by (6.6), $F_{j}(1)$ is a factor of $T_{j}$ for all $t \geq 1 / 2$.

For $d_{j}$ small enough such that (6.8) is positive, $P\left(d_{j}\right)>0$. As we show below, when (6.8) is positive, $H_{j}(t)>0$ for all $t$ so that $T_{j} \rightarrow 0$ as $j \rightarrow \infty$, by (i), and $T_{j}$ does not oscillate.

Now, we complete the proof of conclusion (b) by verifying the essential features of Figure 6.1 and the remarks just discussed. To show (i) for $0<b<1$, first observe that the denominator of $P\left(d_{j}\right)$ is positive since $b \pi^{2} \exp \left(d_{j} / 2\right)>b \pi d_{j}$. Also, for simplicity, we define constants $A, B$, and $C$ such that

$$
P\left(d_{j}\right)=\left[\frac{\left[d_{j}^{2}+A\right] e^{-d_{j} / 2}-C d_{j}}{\left[d_{j}^{2}+B\right] e^{d_{j} / 2}-C d_{j}}\right]\left[\frac{\left[d_{j}^{2}+B\right] e^{-d_{j} / 2}+C d_{j}}{\left[d_{j}^{2}+A\right] e^{d_{j} / 2}+C d_{j}}\right] .
$$

If $P\left(d_{j}\right) \geq 1$, then by clearing the denominator, we have

$$
\left[d_{j}^{2}+A\right]\left[d_{j}^{2}+B\right] e^{-d_{j}} \geq\left[d_{j}^{2}+A\right]\left[d_{j}^{2}+B\right] e^{d_{j}}+2 C d_{j}[B-A] \cosh \left(\frac{d_{j}}{2}\right)
$$

which is clearly impossible as $B>A>0$, and $C, d_{j}>0$ for $0<b<1$. Similarly, if $P\left(d_{j}\right) \leq-1$, then

$$
\begin{aligned}
2 \pi^{2} d_{j}^{2} & \leq[A+B] d_{j}^{2} \\
& \leq\left[d_{j}^{2}+A\right]\left[d_{j}^{2}+B\right] \cosh \left(d_{j}\right)+C d_{j}[B-A] \sinh \left(\frac{d_{j}}{2}\right) \\
& \leq\left(C d_{j}\right)^{2} \leq \pi^{2} d_{j}^{2}
\end{aligned}
$$

which is also clearly impossible.

To show (ii), note that all factors in (6.10) are positive for all $d_{j}>0$ when $0<b<1$ except for the factor (6.8). For small $d_{j}$, (6.8) is positive, but for large $d_{j}$ it is negative. In fact, we can show that there exists exactly one $d_{j}>0$ such that (6.8) is zero. We do this by setting (6.8) equal to zero and solving for $b$, instead of solving for $d_{j}$, to obtain

$$
b=\frac{d_{j}^{2}+\pi^{2}}{d_{j} \pi e^{d_{j} / 2}+\pi^{2}}<1 .
$$

Since the function

$$
f(x)=\frac{x^{2}+\pi^{2}}{x \pi e^{x / 2}+\pi^{2}}
$$

is differentiable (continuous) and strictly decreasing for all $x \geq 0$, and since $f(0)=1$ and $\lim _{x \rightarrow \infty} f(x)=0$, there exists exactly one $d_{j}$ satisfying (6.14) for any given $b$ such that $0<b<1$. Hence, there exists exactly one $d_{j}$, call it $d_{j}^{*}$, making (6.8) zero, and $P\left(d_{j}^{*}\right)=0$.

The fact that $H_{j}(t)>0$ when (6.8) is positive was needed to show that $T_{j}$ did not oscillate for $j$ such that $d_{j}<d_{j}^{*}$, i.e., when $P\left(d_{j}\right)>0$. To show this, we discuss two cases, $n$ odd and $n$ even. 
When $n$ is odd, $H_{j}(t)>0$ for all $d_{j}>0$, regardless of the value of $b>0$. Clearly, $H_{j}(t)>0$ on $[n, n+1 / 2]$ as all terms are positive. So, we must discuss the interval $[n-1 / 2, n]$. On this interval, the cosine term in (6.7) is nonnegative, so we need be concerned only with the sine term. When $d_{j} \leq \pi$, we have

$$
\left[d_{j}^{2}+\pi^{2}(1+b)\right] e^{-d_{j}(t-n)}>\pi^{2} b \geq b d_{j} \pi \geq b d_{j} \pi \sin (\pi t) \geq 0,
$$

so that $H_{j}(t)>0$. When $d_{j}>\pi$, and on $[n-1 / 4, n]$,

$$
-b d_{j}^{2} \cos (\pi t)>b d_{j} \pi \sin (\pi t)
$$

so that $H_{j}(t)>0$ on $[n-1 / 4, n]$. On $[n-1 / 2, n-1 / 4]$, regardless of the value of $d_{j}>0$,

$$
\left[d_{j}^{2}+\pi^{2}(1+b)\right] e^{-d_{j}(t-n)}>\pi^{2} b e^{d_{j} / 4}>b d_{j} \pi \sin (\pi t),
$$

so that $H_{j}(t)>0$ on $[n-1 / 2, n-1 / 4]$, completing the proof of our claim for $n$ odd.

When $n$ is even and $0<b<1$, we again consider the intervals $[n-1 / 2, n]$ and $[n, n+1 / 2]$ separately. On $[n-1 / 2, n]$, only the cosine term is negative. Since this term is dominated by the exponential term, i.e.,

$$
\left[d_{j}^{2}+\pi^{2}(1-b)\right] e^{-d_{j}(t-n)}>d_{j}^{2}>b d_{j}^{2} \cos (\pi t),
$$

we have $H_{j}(t)>0$ on $[n-1 / 2, n]$. When $d_{j}<d_{j}^{*}$, (6.8) is strictly positive as noted above. This allows us to show that $H_{j}(t)$ is strictly decreasing on $[n, n+1 / 2]$ as

$$
\begin{aligned}
H_{j}^{\prime}(t) & =\frac{-d_{j}\left\{\left[d_{j}^{2}+\pi^{2}(1-b)\right] e^{-d_{j}(t-n)}-b d_{j} \pi \sin (\pi t)\right\}-b d_{j} \pi^{2} \cos (\pi t)}{\left(d_{j}^{2}+\pi^{2}\right)(1-b)} \\
& \leq \frac{-d_{j}\left\{\left[d_{j}^{2}+\pi^{2}(1-b)\right] e^{-d_{j} / 2}-b d_{j} \pi\right\}-b d_{j} \pi^{2} \cos (\pi t)}{\left(d_{j}^{2}+\pi^{2}\right)(1-b)}<0 .
\end{aligned}
$$

Again, by the fact that (6.8) is positive for $d_{j}<d_{j}^{*}$,

$$
\lim _{t \rightarrow(n+1 / 2)^{-}} H_{j}(t)>0,
$$

so that $H_{j}(t)>0$ on $[n, n+1 / 2]$. This completes the proof of our claim that $H_{j}(t)>0$ for all $t$ when $j$ satisfies $d_{j}<d_{j}^{*}$.

One additional conclusion that we can draw from (6.14) is that $P\left(d_{j}\right)=0$ only when $|b|<1$. Here, we have made use of the symmetry remark presented at the beginning of the proof.

Finally, because of the exponential factors $\exp \left(d_{j} / 2\right)$ in the denominator of $P\left(d_{j}\right)$, we see that

$$
\lim _{d_{j} \rightarrow \infty} P\left(d_{j}\right)=0
$$

which, when combined with (ii), is (iii). Furthermore, $T_{j} \rightarrow 0$ rapidly for large $d_{j}$ as $P\left(d_{j}\right) \approx 0$. 
Conclusion (c). To prove conclusion (c), we take the same approach as used for conclusion (b). We graph $P\left(d_{j}\right)$ as a function of $d_{j}$, explore the graph's relevant features, explain how these features dictate the behavior of the solutions $T_{j}$, and then show why the graph of $P\left(d_{j}\right)$ must have the features described.

The graph of $P\left(d_{j}\right)$ as a function of $d_{j}$ has two general forms depending on $b>1$. If $b>1$ is small, $P\left(d_{j}\right)$ looks like the curve in Figure 6.2 while if $b>1$ is large, $P\left(d_{j}\right)$ appears as in Figure 6.3. Note the following similarities in these two graphs

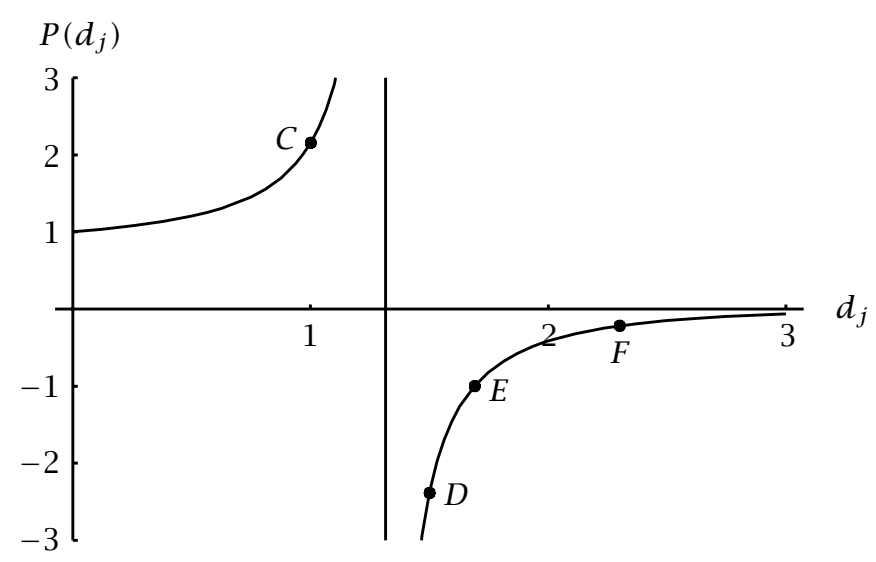

FIGURE 6.2. Product ratio $P\left(d_{j}\right)$ for $b(t)=b \cos (\pi t),|b|>1$ and small.

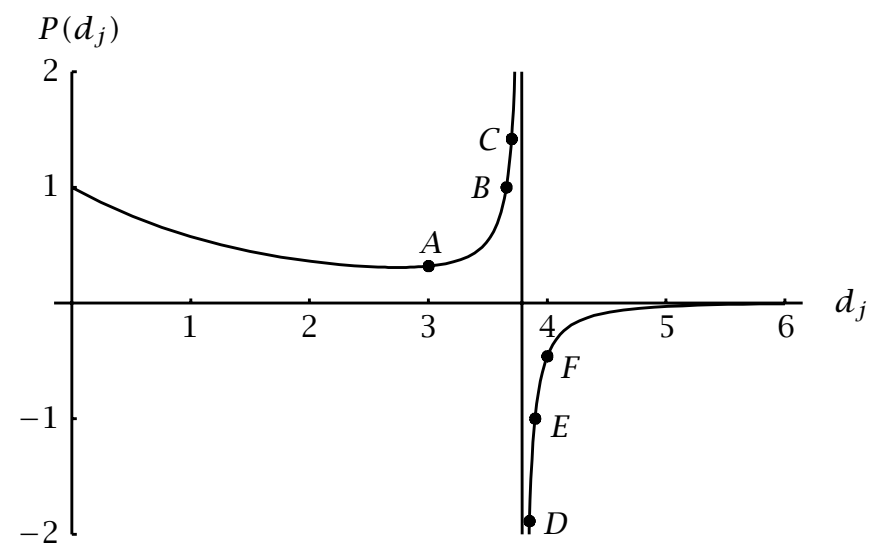

FIGURE 6.3. Product ratio $P\left(d_{j}\right)$ for $b(t)=b \cos (\pi t),|b|>1$ and large.

(i) $P\left(d_{j}\right)$ has a vertical asymptote located at some value $d_{j}^{*}>0$.

(ii) $P\left(d_{j}\right)$ is negative for $d_{j}>d_{j}^{*}$ and approaches the axis from below as $t \rightarrow \infty$, but never crosses the time axis.

(iii) $P\left(d_{j}\right)$ is positive for $0<d_{j}<d_{j}^{*}$ and $P\left(d_{j}\right) \rightarrow \infty$ as $d_{j}$ approaches $d_{j}^{*}$ from the left. 
The difference in the two graphs is that for large $b$ (Figure 6.3), $P\left(d_{j}\right)$ decreases for a short period on the interval $0<d_{j}<d_{j}^{*}$ before increasing while for $b$ small (Figure 6.2), $P\left(d_{j}\right)$ is strictly increasing on $0<d_{j}<d_{j}^{*}$. The significance of this difference is that for $b$ large, the product ratio $P\left(d_{B}\right)=1$ for some $d_{B}$ satisfying $0<d_{B}<d_{j}^{*}$ (See point B on Figure 6.3). This means that the corresponding solutions $T_{j}$ are periodic with period 2 (see Figure 6.4). The period must be 2 because $H_{j}(t)$ is 2-periodic for all $j$ and because the product of any two consecutive factors $F_{j}(n)$ and $F_{j}(n+1)$ in (6.6) is 1 . For $b>1$ small (Figure 6.2), we do not have this type of solution because $P\left(d_{j}\right) \neq 1$ for all $d_{j}>0$.

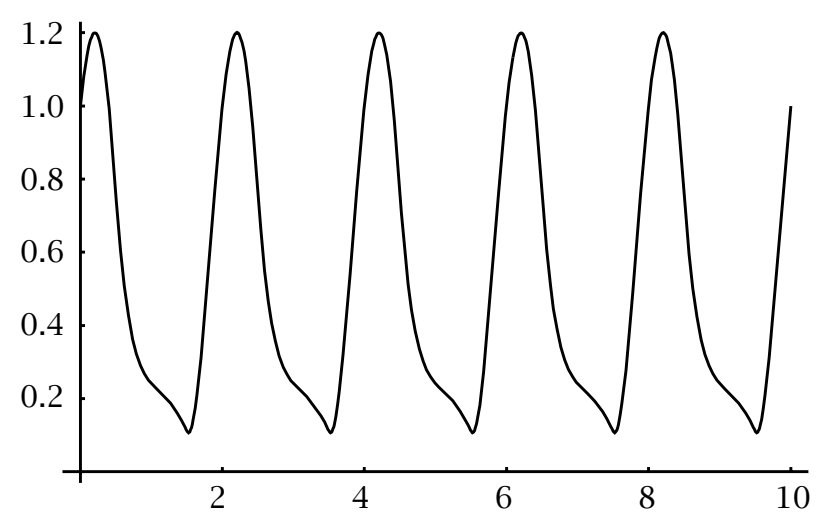

FIGURE 6.4. $T_{j}(t)$ for $b(t)=b \cos (\pi t), b=3, d_{j}=3.656$.

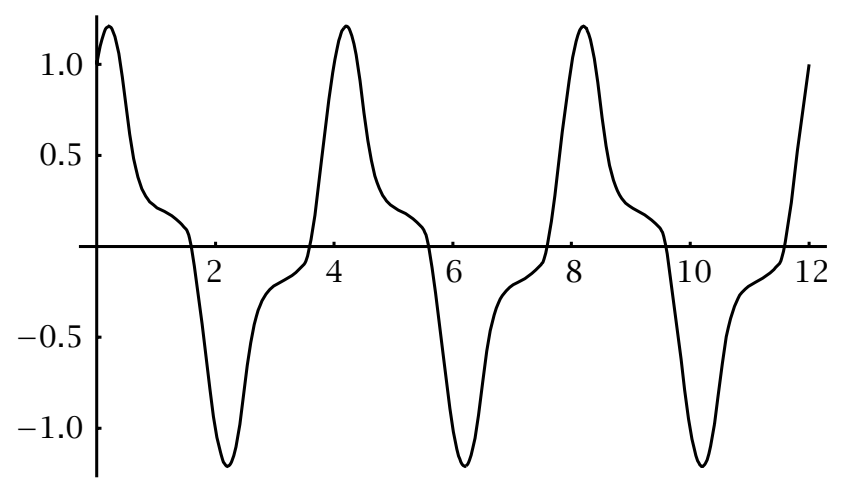

FIGURE 6.5. $T_{j}(t)$ for $b(t)=b \cos (\pi t), b=3, d_{j}=3.897$.

The other interesting feature, which is present in both Figures 6.2 and 6.3 (see point E on those graphs), is that $P\left(d_{E}\right)=-1$ for some $d_{E}>d_{j}^{*}$. The corresponding $T_{j}$ solutions must oscillate and are 4-periodic (see Figure 6.5). This follows from the fact that $H_{j}(t)$ is 2-periodic and that $P\left(d_{E}\right)^{2}=F_{j}(n) F_{j}(n+1) F_{j}(n+2) F_{j}(n+3)=1$ for any integer $n=1,2,3, \ldots$ so that $T_{j}(t+4)=T_{j}(t)$ (see (6.6)). 


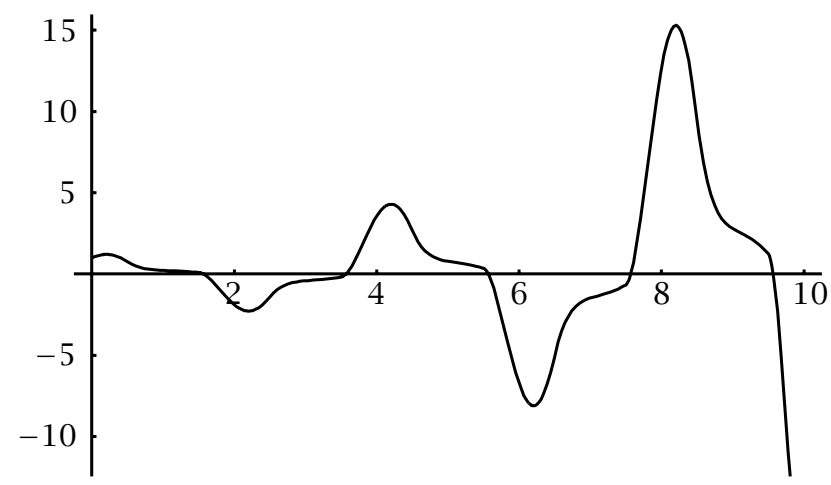

FIGURE 6.6. $T_{j}(t)$ for $b(t)=b \cos (\pi t), b=3, d_{j}=3.85$.

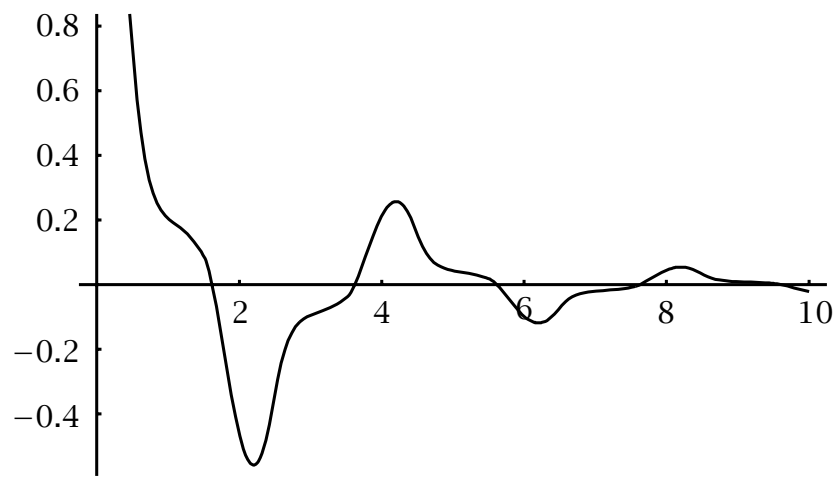

FIGURE 6.7. $T_{j}(t)$ for $b(t)=b \cos (\pi t), b=3, d_{j}=4$.

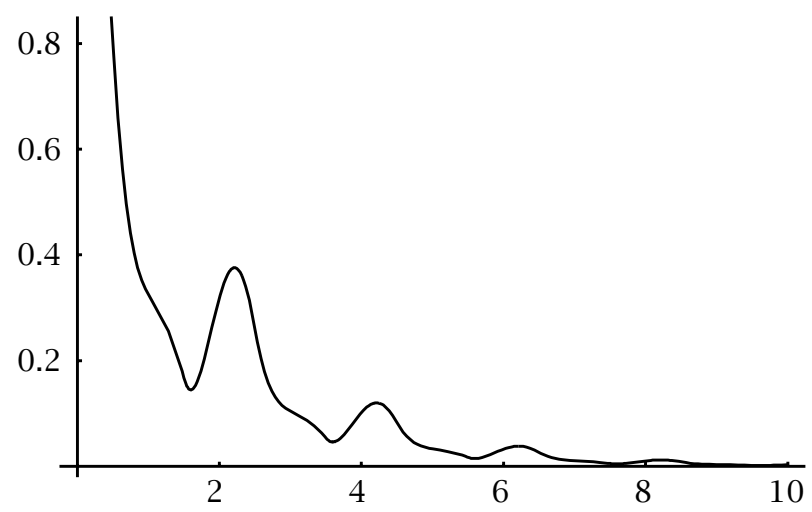

FIGURE 6.8. $T_{j}(t)$ for $b(t)=b \cos (\pi t), b=3, d_{j}=3$.

We see other types of behaviors for $T_{j}$ as well. For example, $P\left(d_{D}\right)<-1$ for $d_{D}$ slightly larger than $d_{j}^{*}$ so that the solutions $T_{j}$ exhibit growing oscillations (see Figure 


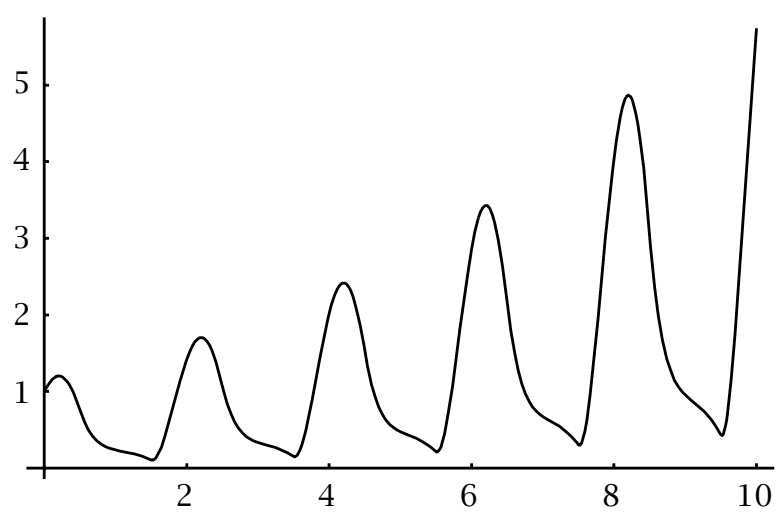

FIGURE 6.9. $T_{j}(t)$ for $b(t)=b \cos (\pi t), b=3, d_{j}=3.7$.

6.6) while for $d_{F}$ much larger than $d_{j}^{*},-1<P\left(d_{F}\right)<0$ (see $\mathrm{F}$ in Figures 6.2 and 6.3) so that $T_{j}$ possesses damping oscillations (see Figure 6.7). Note that the 4-periodic solution at $d_{E}$ separates these two behaviors, that is, for $d_{j}$ satisfying $d_{j}^{*}<d_{j}<d_{E}$, we have growing oscillations while, for $d_{j}$ satisfying $d_{E}<d_{j}$, we have decaying oscillations. The 4-periodic solution serves as the dividing line between these two types of solutions.

Similarly, in Figure 6.3, we see that for $b>1$ and large, the 2-periodic solution at $d_{B}$ (point B) separates dampening solutions for all $d_{A}$ such that $0<d_{A}<d_{B}$ from growing solutions for all $d_{C}$ such that $d_{B}<d_{C}<d_{j}^{*}$. See Figures 6.8 and 6.9. For $b>1$ and small (Figure 6.2), we see that $P\left(d_{j}\right)$ is strictly increasing for $d_{j}$ satisfying $0<d_{j}<d_{j}^{*}$ so that we have solutions $T_{j}$ which grow as $t \rightarrow \infty$.

Now, we explain why the graphs of the product ratio $P\left(d_{j}\right)$ are as they appear in Figures 6.2 and 6.3. We begin by discussing the sign of the product ratio written-out in (6.10). The two factors containing the term $\pi^{2}(1+b)$ are positive for all $d_{j}>0$. (Note that $\exp \left(d_{j} / 2\right)>d_{j}$.) The factor in the numerator containing the term $\pi^{2}(1-b)$ is negative for all $d_{j}>0$ since $d_{j} \exp \left(-d_{j} / 2\right)<2<\pi$. The factor

$$
\left[d_{j}^{2}+\pi^{2}(1-b)\right] e^{d_{j} / 2}+b d_{j} \pi
$$

in the denominator is negative for $d_{j}>0$ small and positive for $d_{j}$ large. To see this, note that this factor is approximately $\pi^{2}(1-b)<0$ for $d_{j}$ near 0 while, for $d_{j}>\pi \sqrt{b-1}$, it is positive. Since (6.23) is continuous, there exists at least one $d_{j}^{*}$ such that (6.23) is zero. In fact, there is only one such $d_{j}^{*}$ because once (6.23) becomes positive, it stays positive. The proof of this fact is lengthy and is given at the end of this section in Lemma 6.2. Combining the information of the previous paragraph, we see that $P\left(d_{j}\right)>0$ for $0<d_{j}<d_{j}^{*}$ and $P\left(d_{j}\right)<0$ for $d_{j}>d_{j}^{*}$. Furthermore, $P\left(d_{j}^{*}\right)$ is not defined as (6.23) is zero so that we have a vertical asymptote at $d_{j}^{*}$. In fact, if we set $x=d_{j}^{*}$ in (6.23) and solve for $b$, we get

$$
b=g(x) \equiv \frac{x^{2}+\pi^{2}}{\pi\left(\pi-x e^{-x / 2}\right)}>1 .
$$


The function $g$ is well defined and is larger than 1 because $x e^{-x / 2}<2$ for all $x>0$. This says that we can have a vertical asymptote for $b>1$ only. Furthermore, since $g$ is continuous for all $x \geq 0, g(0)=1$, and $\lim _{x \rightarrow \infty} g(x)=\infty$, we have a vertical asymptote for any given value of $b>1$. Finally, as noted in the discussion of conclusion (b), $P\left(d_{j}\right) \neq 0$ for all $d_{j}>0$ when $b>1$ because the factors in the numerator are never zero.

Now, the properties (i), (ii), and (iii) of the graphs in Figures 6.2 and 6.3 follow from these remarks and the fact that

$$
\lim _{d_{j} \rightarrow \infty} P\left(d_{j}\right)=0
$$

which is a consequence of the exponentials in the denominator.

The last detail to attend to is to show the uniqueness of the zero $d_{j}^{*}$ of (6.23). This is Lemma 6.2 which follows.

LEMMA 6.2. For $b>1$, the function

$$
f(x) \equiv\left[x^{2}+\pi^{2}(1-b)\right] e^{x / 2}+b \pi x
$$

has exactly one zero on the interval $x \geq 0$.

Proof. Instead of $f(x)$, we consider the related function

$$
F(x) \equiv e^{-x} f(2 x)=4 x^{2}+\pi^{2}-b \pi\left[\pi-2 x e^{-x}\right] .
$$

Note that if $d_{j}^{*}$ is a zero of $f(x)$, then $x^{*}=d_{j}^{*} / 2$ is a zero of $F(x)$, and vice versa. Furthermore, we consider the two separate, continuous functions

$$
F_{1}(x)=4 x^{2}+\pi^{2}, \quad F_{2}(x)=b \pi\left[\pi-2 x e^{-x}\right],
$$

which are graphed in Figure 6.10 and which intersect at the zeros of $F(x)$. Note that $F_{2}(x) \leq b \pi^{2}$ for all $x \geq 0$ whereas $F_{1}(x)$ is a parabola which grows without bound. Furthermore, since $F_{1}(0)=\pi^{2}<b \pi^{2}=F_{2}(0)$, the graphs of the functions $F_{1}$ and $F_{2}$ must cross at least once on the interval $x \geq 0$. To show that there is only one intersection point, we need the following observations, which the reader can verify easily by computing derivatives and considering Figure 6.10 .

(1) $F_{2}^{\prime}(x) \leq F_{2}^{\prime}$ (2) for all $x \geq 0$ and $F_{2}^{\prime}(x)$ is decreasing for $x \geq 2$.

(2) $F_{1}^{\prime}(0)=0$ and $F_{1}^{\prime}(x)$ is increasing for all $x \geq 0$.

(3) $F_{2}(1) \leq F_{2}(x) \leq F_{2}(0)=b \pi^{2}$ for all $x \geq 0$.

With these facts, we show that once $F_{1}(x)$ and $F_{2}(x)$ intersect at some point $x^{*} \geq 0$, $F_{1}$ and $F_{2}$ do not intersect again as $F_{1}(x)$ increases more rapidly than $F_{2}(x)$ for all $x \geq x^{*}$. We do this by considering two separate cases: $1<b \leq 5$ and $b>5$.

CASE $1<b \leq 5$. For $0 \leq x \leq 1, F_{2}^{\prime}(x)<0 \leq F_{1}^{\prime}(x)$. For $x \geq 1$, by (1) and (2) above,

$$
F_{2}^{\prime}(x) \leq F_{2}^{\prime}(2) \equiv 2 \pi b e^{-2} \leq 2 \pi 5 e^{-2}<8=F_{1}^{\prime}(1) \leq F_{1}^{\prime}(x) .
$$

Hence, for all $x \geq 0, F_{2}^{\prime}(x)<F_{1}^{\prime}(x)$ so that once $F_{1}$ and $F_{2}$ intersect, they do not intersect again as $F_{1}$ grows more quickly than $F_{2}$. 


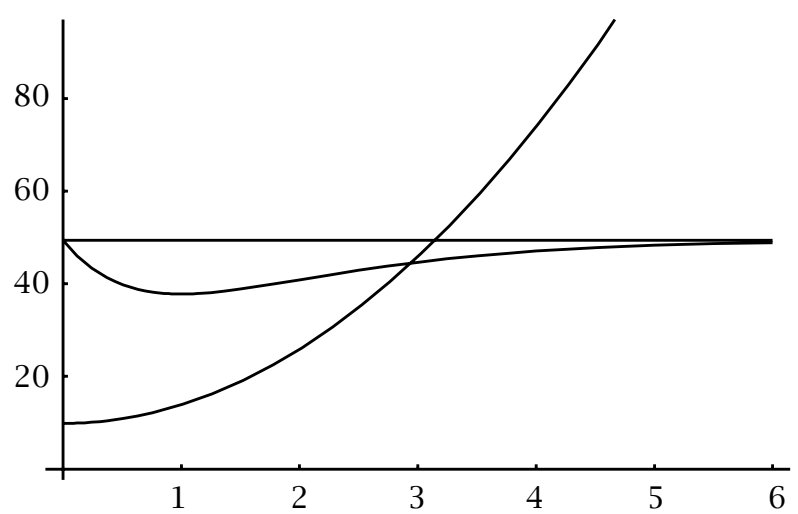

FigURE 6.10. $F_{1}(x), F_{2}(x)$, and $y=b \pi^{2}$ for $b=5$.

CASE $b>5$. We must be more careful in this case. First, we note that because the range of $F_{2}(x)$ is bounded by $F_{2}(1)$ and $F_{2}(0)$ (see (3) above), $F_{1}(x)$ and $F_{2}(x)$ intersect only at those values of $x$ for which $F_{1}(x)$ is likewise bounded by $F_{2}(1)$ and $F_{2}(0)$, i.e.,

$$
b \pi(\pi-1)<b \pi\left(\pi-2 e^{-1}\right)=F_{2}(1) \leq F_{1}(x) \leq F_{2}(0)=b \pi^{2} .
$$

Solving this for $x$, we see that the $x$-coordinate of any intersection point must satisfy

$$
\sqrt{\frac{b(\pi-1)-\pi}{\pi}} \frac{\pi}{2}<x \leq \sqrt{b-1} \frac{\pi}{2} .
$$

Therefore, for all $b>5$, all intersection points must occur on the interval

$$
\sqrt{\frac{5(\pi-1)-\pi}{\pi}} \frac{\pi}{2} \approx 2.4 \leq x .
$$

Since $F_{2}^{\prime}(x)$ is decreasing on $x \geq 2$, by (1) above, the largest value of $F_{2}^{\prime}(x)$ on the interval (6.31) must occur at the left endpoint, which we denote by the function

$$
L(b) \equiv \sqrt{\frac{b(\pi-1)-\pi}{\pi}} \frac{\pi}{2} \geq 2.4 .
$$

Computing the following estimates, we have

$$
L(b) \geq \sqrt{\frac{b(\pi-1)-b}{\pi}} \frac{\pi}{2}=\frac{\sqrt{b} \sqrt{\pi-2} \sqrt{\pi}}{2} \geq \frac{\sqrt{\pi}}{2} \sqrt{b},
$$

and

$$
L(b) \leq \sqrt{\frac{b \pi}{\pi}} \frac{\pi}{2}=\sqrt{b} \frac{\pi}{2} .
$$

Hence, since the largest value of $F_{2}^{\prime}(x)$ on the interval (6.31) occurs at the left endpoint,

$$
\begin{aligned}
F_{2}^{\prime}(L(b)) & =b 2 \pi e^{-L(b)}[L(b)-1] \\
& \leq b 2 \pi e^{(-\sqrt{\pi b} / 2)}\left[\sqrt{b} \frac{\pi}{2}\right] \\
& =\sqrt{b} \pi^{2} g(b),
\end{aligned}
$$


where a simple calculation shows that

$$
g(b)=b e^{-\sqrt{\pi b} / 2}
$$

has a maximum at $b=16 / \pi$, which is

$$
g(b) \leq g\left(\frac{16}{\pi}\right)=\frac{16}{\pi} e^{-2} .
$$

Therefore, for all $x$ satisfying (6.31), we have

$$
F_{2}^{\prime}(x) \leq F_{2}^{\prime}(L(b)) \leq \sqrt{b} \pi^{2}\left(\frac{16}{\pi} e^{-2}\right) \leq 6.81 \sqrt{b} .
$$

On the other hand, since $F_{1}^{\prime}(x)$ is increasing for all $x \geq 0$, by (2), the minimum of $F_{1}^{\prime}(x)$ for $x \geq L(b)$ follows from

$$
F_{1}^{\prime}(x) \geq F_{1}^{\prime}(L(b))=8 L(b) \geq 8 \frac{\sqrt{\pi}}{2} \sqrt{b}=4 \sqrt{\pi} \sqrt{b} \geq 7 \sqrt{b} .
$$

Hence, for $b>5$ and for all $x$ on the interval (6.31), $F_{2}^{\prime}(x) \leq 6.81 \sqrt{b}<7 \sqrt{b} \leq F_{1}^{\prime}(x)$ so that once $F_{1}$ and $F_{2}$ intersect, they do not intersect again as $F_{1}$ grows more rapidly than $F_{2}$. This completes the proof of Lemma 6.2.

7. A scalar EPCA for $b(t)=b e^{s t}$. The behavior of the time solutions $T_{j}(t)$ are very complicated for $b(t)=b e^{s t}$. Not only must we deal with five parameters $a, b, j, n$, and $s$ in attempting to analyze the solutions, but, as we see below, the ratio function $F_{j}(n)$ does indeed depend on $n$ in a nontrivial way. Recall that for $b(t)=b$ and $b(t)=b \cos (2 \pi t), F_{j}(n)$ is independent of $n$, i.e., constant for $j$ fixed while, for $b(t)=$ $b \cos (\pi t), F_{j}(n)$ depends only on whether $n$ is even or odd. In the present case, $F_{j}(n)$ varies with $n=[t+1 / 2]$ so that the solutions $T_{j}(t)$ may change behavior as $t$ increases. For example, $F_{j}(n)$ may be greater than 1 for the first few values $n=1,2,3, \ldots$ meaning that the solutions grow without oscillation. Then for the next few values of $n, F_{j}(n)$ becomes less than -1 meaning that solutions grow with oscillation, then the solutions dampen with oscillation as $-1<F_{j}(n)<0$ for larger values of $n$, and finally $T_{j} \rightarrow 0$ monotonically as $F_{j}(n)$ satisfies $0<F_{j}(n)<1$ for all large $n$. Therefore, to catalog all solution behavior, as was done for the cases $b(t)=b$ and $b(t)=b \cos (2 \pi t)$, is exceedingly difficult, if not impossible and probably not interesting. Therefore, we concentrate only on the asymptotic behavior of the solutions $T_{j}$ which can be readily classified by comparing the parameters $s$ and $d_{j}=a^{2} \pi^{2} j^{2}$. The interesting behavior occurs for $s=d_{j}$ where the corresponding solution $T_{j}$ approaches a periodic curve which is not itself a solution to the differential equation (4.6). In the previous examples, the periodic solutions were all solutions to the differential equation (4.6) for all time $t \geq 0$.

First, we write the analytic formulas for the $T_{j}$ solutions. By (4.22), the functions $E_{n j}$ become

$$
E_{n j}(t)= \begin{cases}\frac{b d_{j} e^{s t}}{\left(s+d_{j}\right)\left(b e^{s n}-1\right)}, & \text { if } s+d_{j} \neq 0, \\ \frac{d_{j} e^{-d_{j} t} b t}{b e^{-d_{j} n}-1}, & \text { if } s+d_{j}=0,\end{cases}
$$


provided $b e^{s n} \neq 1$ for all $n=1,2,3, \ldots$. The solutions $T_{n j}$ follow from (7.1) and equations (4.21), (4.22), (4.23), and (4.24).

THEOREM 7.1. The solution to BVP (4.1), with $b(t)=b e^{\text {st }}$ and with be $e^{\text {sn }} \neq 1$ for all $n=1,2,3, \ldots$, has time functions $T_{j}(t)$ given by (4.21), (4.22), (4.23), (4.24) and (7.1). If $F_{j}(n)$ is defined and nonzero for all $n=1,2,3, \ldots$, the solutions $T_{j}$ behave as follows

(a) For $s<0$, the functions $T_{j}(t) \rightarrow 0$ as $t \rightarrow \infty$ with the approach being monotone for $t$ large enough.

(b) For $s=0, b(t)=b$, which we have already discussed in Section 3.

(c) For $0<s<d_{j}$, the functions $T_{j}(t) \rightarrow 0$ as $t \rightarrow \infty$, but the convergence is not monotone.

(d) For $s=d_{j}$, as $t \rightarrow \infty$, the solutions $T_{j}$ approach the periodic curve $C_{j} \cosh (s(t-$ $n)$ ), which is not a solution to the differential equation (4.6).

(e) For $0<d_{j}<s, T_{j}(t)$ grows without bound and without oscillation for $t$ large enough, but the growth is not monotone.

If $F_{j}(k)=0$ for some positive integer $k$, then $T_{j}(t)=0$ for $t \geq k-1 / 2$ as can be seen from (4.29). If condition (4.26) is violated so that $F_{j}(k)$ is not defined for some positive integer, then $u_{0}(x)$ must be orthogonal to $\sqrt{2} \sin (\pi j x)$ as described in the second remark following Theorem 4.1, and $T_{j}(t)=0$ for all $t \geq 0$. On the other hand, if $u_{0}$ is not orthogonal to $\sqrt{2} \sin (\pi j x)$, our method does not provide a solution.

Proof. We consider several cases.

CASE (a) $s<0$. Observe that as $t \rightarrow \infty, n=[t+1 / 2] \rightarrow \infty$, and $E_{n j}(t) \rightarrow 0$ so that $D_{n j}=1-E_{n j}(n) \rightarrow 1$. Hence, $H_{j}(t) \equiv D_{n j} e^{-d_{j}(t-n)}+E_{n j}(t)$ approaches the 1-periodic function $e^{-d_{j}(t-n)}$. (Note that this is periodic because the difference $t-n$ is periodic.) Also,

$$
F_{j}(n)=\frac{D_{(n-1) j} e^{-d_{j} / 2}+E_{(n-1) j}(n-0.5)}{D_{n j} e^{d_{j} / 2}+E_{n j}(n-0.5)} \longrightarrow \frac{1}{e^{d_{j}}},
$$

and since $d_{j}>0$, we see that as $n \rightarrow \infty$,

$$
\prod_{k=1}^{n} F_{j}(k) \longrightarrow 0
$$

Hence, $T_{j}$ goes to zero as $t$ approaches infinity if $s<0$. This decay is monotone for $t$ large enough as $e^{-d_{j}(t-n)}$ decreases on each interval $[n-1 / 2, n+1 / 2), n=1,2,3, \ldots$. We remark that the time solution to the nondelay equation is

$$
T_{j}(t)=C_{j}\left(1-b e^{s t}\right)^{d_{j} / s} e^{-d_{j} t}
$$

which also approaches zero as $t \rightarrow \infty$. This formula is valid whenever $1-b \exp (s t)$ is positive, or if it is negative, then $d_{j} / s$ is such that the function $p(t)=p^{d_{j} / s}$ is defined for $p<0$, e.g., if $s$ is an odd integer or if $d_{j}$ is an even integer.

CASE (b) $0<s<d_{j}$. For $t$ large, $E_{n j}(t)$ approaches the function

$$
\frac{d_{j}}{s+d_{j}} e^{s(t-n)}
$$


and

$$
D_{n j}=1-E_{n j}(n) \longrightarrow \frac{s}{s+d_{j}} .
$$

Hence, $H_{j}(t)$ approaches the bounded, periodic function

$$
\frac{s e^{-d_{j}(t-n)}+d_{j} e^{s(t-n)}}{s+d_{j}}=\frac{e^{-d_{j}(t-n)}}{s+d_{j}}\left[s+d_{j} e^{\left(s+d_{j}\right)(t-n)}\right]
$$

for $t$ large. Also, as $t \rightarrow \infty, n \rightarrow \infty$ and so

$$
F_{j}(n) \rightarrow \frac{s e^{-d_{j} / 2}+d_{j} e^{s / 2}}{s e^{d_{j} / 2}+d_{j} e^{-s / 2}} .
$$

For $0<s<d_{j}$, this ratio is positive but less than 1 . To see this, realize that the function

$$
f(x)=\frac{\sinh (x / 2)}{x}
$$

is increasing for $x \geq 0$ (the Maclaurin series for $f$ may be helpful when verifying this), and proceed as below. Since $0<s<d_{j}$,

$$
\begin{gathered}
\frac{\sinh (s / 2)}{s}<\frac{\sinh \left(d_{j} / 2\right)}{d_{j}} \\
\frac{e^{s / 2}-e^{-s / 2}}{s}<\frac{e^{d_{j} / 2}-e^{-d_{j} / 2}}{d_{j}} \\
s e^{-d_{j} / 2}+d_{j} e^{s / 2}<s e^{d_{j} / 2}+d_{j} e^{-s / 2} \\
\frac{s e^{-d_{j} / 2}+d_{j} e^{s / 2}}{s e^{d_{j} / 2}+d_{j} e^{-s / 2}}<1 .
\end{gathered}
$$

Consequently, as $n \rightarrow \infty$,

$$
\prod_{k=1}^{n} F_{j}(k) \longrightarrow 0
$$

and, therefore, $T_{j}(t)$ approaches 0 as $t$ goes to infinity. However, this decay is not monotone as (7.7) is decreasing on $[n-1 / 2,0)$, but is increasing on $[0, n+1 / 2)$, as the reader can verify. Note also that the limit of the ratio $F_{j}(n)$ is independent of $b$.

For the nondelay equation,

$$
T_{j}(t) \rightarrow C_{j}(-b)^{d_{j} / s}
$$

as $t \rightarrow \infty$. The interesting fact, here, is that while $T_{j}$ for the delay equation goes to 0 , regardless of the value of $b$, the limit as $t \rightarrow \infty$ of $T_{j}$ for the nondelay equation depends on $b$, as well as on $a, j$, and $s$. For the delay equation, $b$ affects only the rate at which $T_{j}$ goes to 0 .

CASE (c) $s=0$. Here, $b(t)=b$ and so the associated solutions have already been discussed in Section 3.

CASE (d) $s=d_{j}$. Since $s>0, H_{j}(t)$ and $F_{j}(n)$ also approach (7.7) and (7.8). $H_{j}(t)$ approaches a 1-periodic function, as before, but the ratio in (7.8) is exactly 1 in this 
case. Hence, as $t$ increases, $T_{j}(t)$ approaches the periodic curve $C_{j} \cosh (s(t-n))$. See (7.7) and Figure 7.1. As the reader can verify, this function is not itself a solution to (4.6). This is to be contrasted with the $T_{j}$ solution to the nondelay equation (7.4), which approaches the constant $C_{j}(-b)$.

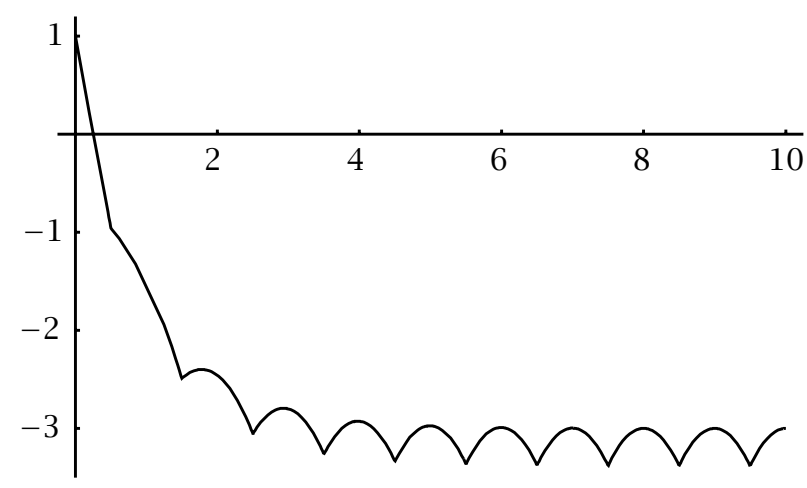

FigURE 7.1. $T_{j}(t)$ for $b(t)=b e^{s t}, b=0.75, s=d_{j}=1, j=1, a=1 / \pi$.

CASE (e) $0<d_{j}<s$. Again (7.7) and (7.8) apply. $H_{j}(t)$ approaches a 1-periodic function, as before, but the ratio in (7.8) is greater than 1 as can be seen by a calculation similar to the one in the case $0<s<d_{j}$. Hence, as $t$ increases, $T_{j}(t)$ grows without bound and without oscillation, for $t$ large enough. The growth is not monotone because (7.7) is alternately decreasing and increasing on each interval $[n-1 / 2, n+1 / 2)$.

Finally, note that $T_{j}$ for the nondelay equation does not grow without bound, but approaches the number $C_{j}(-b)^{d_{j} / s}$ when this value, and, hence, the range values of $T_{j}$ itself are defined as real numbers.

8. Conclusions. We summarize the features of the time solutions $T_{j}$ to the BVP (4.1) and compare these with the time solutions to the nondelay BVP, where the solutions are known.

The most common difference between the time solutions to the delay problem versus the nondelay problem is the "kinks" or discontinuities in the first derivative, that occur at the points $n+1 / 2, n=0,1,2, \ldots$. While the solutions to the nondelay problem may be $C^{\infty}$, which is the case for $b(t)=b$, the delay problems always have discontinuities in the first derivative, producing a "rippling" effect in the solution curves. See, for example, Figures 3.1, 3.3, and 7.1. This phenomenon is caused by the impulsive nature of the delay, and is a familiar feature to researchers working with this type of delay. From the computational standpoint, this discontinuity arises when, in the course of applying the method of steps, we try to splice together the solutions to the time differential equation in adjoining intervals of the form $[n-1 / 2, n+1 / 2]$, $n=0,1,2, \ldots$. Because the solutions to the time differential equation, in each of these intervals, form a one-parameter family, we are able to adjust the parameter to obtain 
continuity of the solutions only, but we are unable to obtain continuity of any of the derivatives.

The "ripples" caused by the discontinuities in the derivatives can produce changes in the nature of the time solution curves as compared to the nondelay equation. One such change is the loss of monotonicity. This is apparent in several of our examples. For instance, for $b(t)=b e^{s t}$ with $s=d_{j}$, the nondelay equation has solution

$$
T_{j}(t)=C_{j}\left(e^{-d_{j} t}-b\right),
$$

which approaches monotonically the constant curve $C_{j}(-b)$. However, the solution to the delay equation approaches the periodic curve $C_{j} \cosh \left(d_{j}(t-n)\right), n=[t+1 / 2]$, but does not do so monotonically. See Figure 7.1.

A more severe effect of the piecewise constant delay is the appearance of oscillations in the solutions where none were present in the solutions to the nondelay equation. For example, for $b(t)=b$, the solutions to the nondelay equations were either unbounded and monotonic, or monotonically decaying to zero. In Figures 3.2 and 3.4, we see that the delay has caused these solutions to oscillate about the time axis.

The most radical difference between the solutions to the delay and nondelay problems is the existence of periodic solutions for the delay problem where such solutions were impossible for the nondelay problem. For example, for $b(t)=b$, all solutions to the nondelay equation either grow monotonically without bound or dampen to zero monotonically, whereas for the delay problem we have the existence of an oscillating, periodic solution (see Figure 3.5). Interestingly, this solution serves to separate the unbounded solutions (small $j$ ) from the bounded, decaying solutions (large $j$ ). See Theorem 3.3(d). Furthermore, the period of the solution is 2, which is double the period of the expression $t-n=t-[t+1 / 2]$ that appears in the analytic solution to all of our examples. See equation (4.29).

The phenomenon of periodic solutions separating growing solutions from decaying solutions is also seen for $b=b \cos (\alpha t)$ for both $\alpha=2 \pi$ and $\alpha=\pi$. For $\alpha=2 \pi$, there are two periodic solutions which arise for different values of the parameters $a, b$, and $j$. See Theorem 5.1. Furthermore, these solutions have different periods. One is of period 1 , which is the same period as of $b(t)=\cos (\alpha t)$ and the expression $t-[t+1 / 2]$, while the other is twice that. For $\alpha=\pi$ and $|b|>1$ large, we also have two periodic solutions that arise for different values of the parameters. See Theorem 6.1. The interesting feature in this case is that the periods are both different from the period of the expression $t-[t+1 / 2]$. One is of period 2 , which is the same as the period of $b(t)=\cos (\pi t)$, but the other is of period 4 , which is twice the period of $b(t)=\cos (\pi t)$.

Finally, the separation of the growing from the dampening solutions is also evident for $b(t)=b e^{s t}$. When $d_{j}<s$, the solutions $T_{j}$ dampen, while for $d_{j}>s$ the solutions $T_{j}$ grow without bound. When $d_{j}=s$, the solution $T_{j}$ approaches the 1-periodic curve $C_{j} \cosh \left(d_{j}(t-n)\right), n=[t+1 / 2]$. This curve is not itself a solution to the delay equation and the solution that approaches it is not itself periodic. See Figure 7.1. Note that this is different from all the previous examples discussed in that the solutions $T_{j}$ were themselves periodic solutions. 


\section{REFERENCES}

[1] J. Wiener, Boundary value problems for partial differential equations with piecewise constant delay, Internat. J. Math. Math. Sci. 14 (1991), no. 2, 363-379. MR 92d:35289. Zbl 749.35061.

[2] J. Wiener and L. Debnath, Boundary value problems for the diffusion equation with piecewise continuous time delay, Internat. J. Math. Math. Sci. 20 (1997), no. 1, 187-195. MR 97m:35268. Zbl 879.35065.

WiENER: DePARTMENT OF MATHEMATICS, UniVersity of TEXAs-PAN AMERICAN, EDinbuRG, TX 78539, USA

E-mail address: jwi ener@math. panam.edu

Heller: Department of Mathematics, University of TeXas-PAN American, EDinburg, TX 78539, USA

E-mail address: he11er@panam. edu 


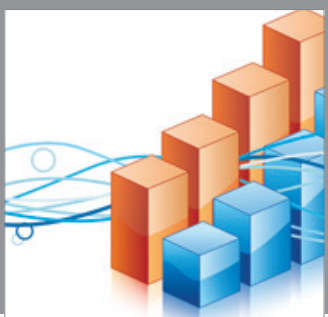

Advances in

Operations Research

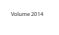

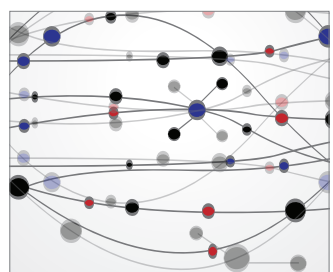

\section{The Scientific} World Journal
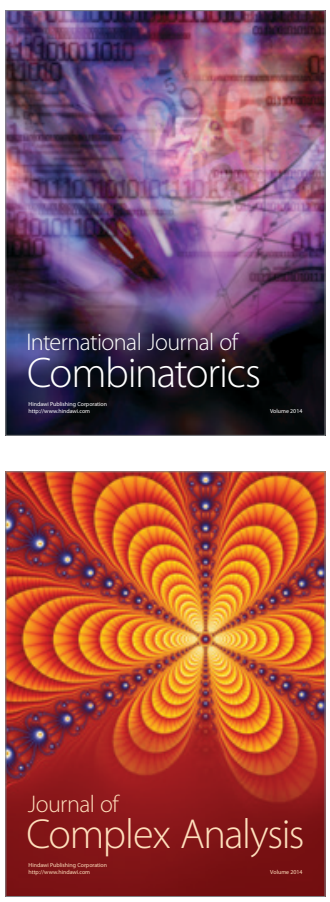

International Journal of

Mathematics and

Mathematical

Sciences
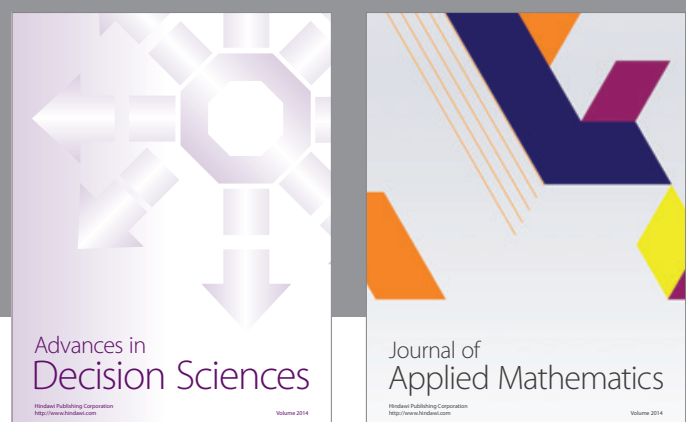

Journal of

Applied Mathematics
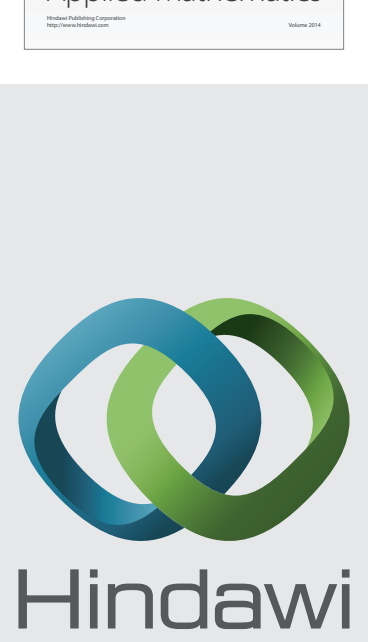

Submit your manuscripts at http://www.hindawi.com
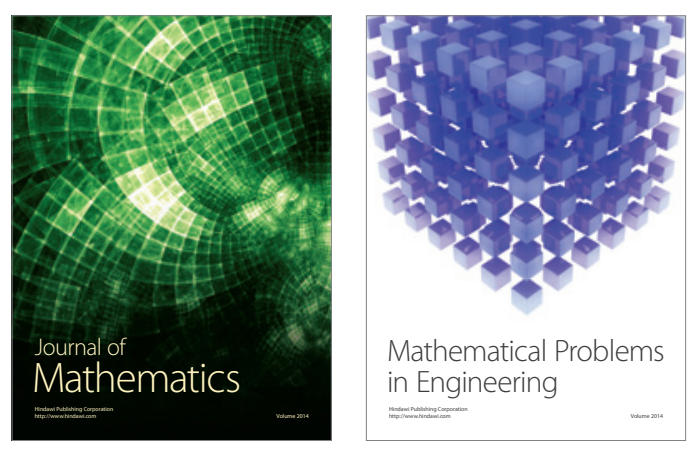

Mathematical Problems in Engineering
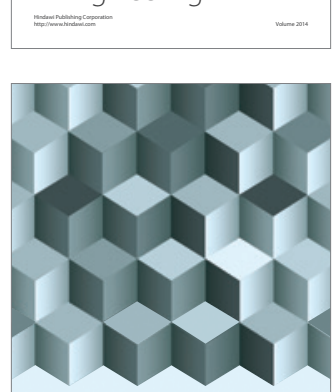

Journal of

Function Spaces
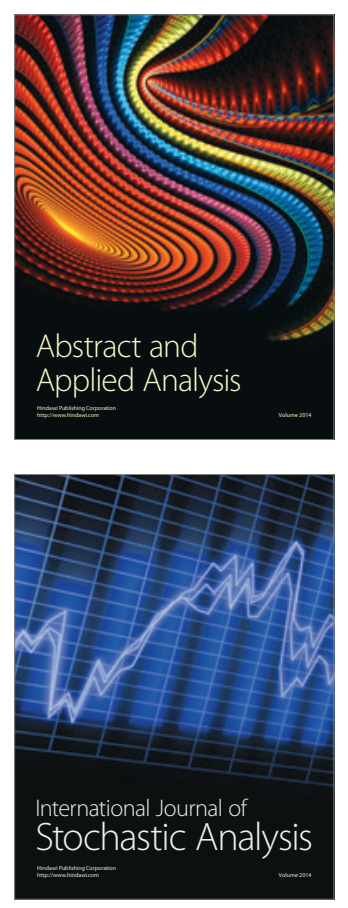

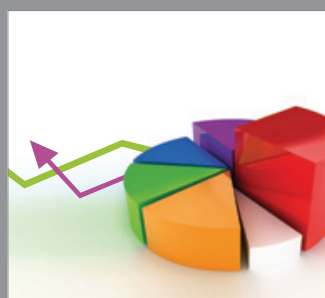

ournal of

Probability and Statistics

Promensencen
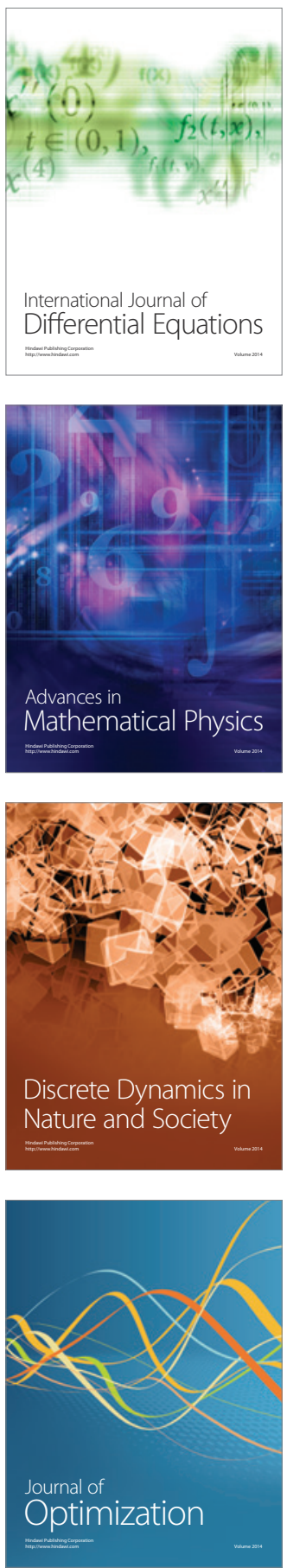